\title{
Optimal Dynamic Pricing of Mobile Data Plans in Wireless Communications
}

\author{
Xiaoyu Ma ${ }^{\mathrm{a}}$, Tianhu Deng ${ }^{\mathrm{b}}$, Mengying Xue ${ }^{\mathrm{b}}$, Zuo-jun Max Shen ${ }^{\mathrm{c}}$, Boxiong Lan ${ }^{\mathrm{d}}$ \\ ${ }^{a}$ International Business School, Beijing Foreign Studies University, Beijing 100089, China, \\ maxythu@gmail.com \\ ${ }^{b}$ Department of Industrial Engineering, Tsinghua University, Beijing 100084, China, \\ \{deng13,xmy14\}@mail.tsinghua.edu.cn \\ ${ }^{c}$ Department of Industrial Engineering and Operations Research, University of California, \\ Berkeley, California94720, USA,shen@ieor.berkeley.edu \\ ${ }^{d}$ School of Economics and Management, Tsinghua University, Beijing 100084, China, \\ lanbx@sem.tsinghua.edu.cn
}

\begin{abstract}
With smartphones sweeping the world, customers use much more data service than voice service nowadays. Because mobile data service occupies much more network capacity than voice, wireless network is frequently congested. We build a dynamic cell phone plan control model where the service provider can decide a subset of plans to open at the beginning of each period. New customers can only subscribe open plans. The dynamic plan control problem is a stochastic programming problem where the system state dimension is exponential in the number of plans and periods. In this paper, we analyze the case when the number of customers approaches positive infinity. In this limiting case, we transform the stochastic dynamic programming problem to an equivalent integer linear programming problem. In a case study, we analyze a large mobile service provider in Asia using 10,000 real individual-level data. Sensitivity analysis implies that a mobile service provider whose initial market share is low can benefit more from the dynamic data plan control.
\end{abstract}

Keywords:

dynamic plan control, mobile data plans, congestion effect, stochastic dynamic programming

(C) 2016. This manuscript version is made available under the Elsevier user license http://www.elsevier.com/open-access/userlicense/1.0/ 


\section{Introduction}

In the last decade, the global wireless communication market has grown rapidly. The percentage of mobile subscriptions had increased from approximately $20 \%$ in 2003 to $96.2 \%$ in 2013 , almost five times worldwide. The International Telecommunication Union predicted that subscriptions would pass seven billion early in 2014 . The wireless communications have become essential to day-to-day lives. Meanwhile, as the mobile market is approaching saturation level, the growth of a new market is slowing down. All mobile service providers focus on competing in the existing market. Commercial competition has been increasingly fierce in wireless communications. In this competitive environment, successful pricing and operation is crucial to a mobile service provider.

With smartphone sweeping the world, customers use much more data service than voice service. The voice usage, traditionally the largest part of business for mobile service providers, grows slowly or even declines in recent years as more and more customers use smartphone apps to communicate, (e.g., Google Voice and Skype). Conversely, global mobile data traffic will increase nearly tenfold between 2014 and 2019, reaching 24.3 exabytes per month by 2019 according to Cisco Visual Networking Index: Global Mobile Data Traffic Forecast Update 2014-2019 White Paper. Moreover, mobile data revenue will overtake voice revenue globally by 2018 (predicted by the Global System for Mobile Communications Alliance at 2013 Mobile World Congress). Thus, data service plays a more and more important role in the wireless communications industry. Many mobile service providers are changing their focus to data service. For example, four major U.S. mobile service providers, AT\&T, Verizon, Sprint, and T-mobile, offer "free" voice and message and charge only data usages in "mobile share value plans".

Meanwhile, the characteristic of data service is partially different from traditional voice service. Mobile service providers are using far more network capacity to deliver one dollar's worth of data service than they are using to deliver one dollar's 
worth of voice service. Customers use an increasing number of data service, which occupies much more network capacity than voice service. As a result, an overload of data usage leads to network congestion (Garg, 2010). Because many customers are sensitive to the delay implied by the congestion (Ho and Zheng, 2004), how to price and control mobile data plans is a new challenge.

We analyze the plan management of a large mobile service provider in Asia. This service provider offers a large number of plans (up to 30 plans) from 2011 to 2013 . At the same time, it invests enormously in advertising, promotion, and phone subsidy to attract new customers. As a response, the rate of new customers stays at a high level (around $3.6 \%$ to $8.9 \%$ per month), and data usage increases fast (around $7.5 \%$ to $16.7 \%$ per month). However, to their deep surprise, the revenue grows very slowly or even declines (around - $0.4 \%$ to $1.9 \%$ per month). This is called "volume increases but revenue does not increase" phenomenon in the industry. Meanwhile, the churn rate becomes higher than those at the traditional voice dominated times (around $2.1 \%$ to $5.2 \%$ per month). The churn rate exceeds half of the new customer's add-in rate. A direct consequence is that the net add-in rate stays at a low level. This effect is called the "large inflows and large outflows" phenomenon in the industry. These two phenomena have troubled this service provider for a long time. According to Analysys International consulting agency's survey data, the most important reason leading to high churn rate is the bad customer experience of data network quality. The slow growth of revenue is partially due to low customer satisfaction.

In fact, the service provider spends lots of money to attract new customers but does not fully consider the fact that the data network capacity is fixed and limited. A lot of new customers experience data network congestion, which is reflected as high churn rates of both new and old customers in practice. In this paper, we propose a dynamic control model of cell phone data plans where the service provider can decide a subset of plans to open at the beginning of each period. New customers can only subscribe open plans. This dynamic control allows the service provider 
to dynamically adjust data network utilization and, thus, achieve high customer satisfaction and low churn rate.

Our model tries to capture four important features of wireless data system: (1) finite capacity, (2) customers have different data usages but share the same capacity, (3) no resource pooling when the total data usages of current customers is less than the capacity, and (4) customers may leave the system when the total data usages of current customers is more than the capacity and congestion effect arises. This dynamic control problem is a stochastic programming problem where the system state dimension is exponential in the number of plans and periods. We analyze the limiting case when the number of customers approaches positive infinity and propose an optimal and efficient solution method.

In order to validate our model, we try to contact the large mobile service provider mentioned above. Finally, it provides us a random sample of 10,000 individual customers from March 2012 to February 2013. Our model is applied to optimize pricing for this company based on the data. The results show that dynamic control can help the company increase revenue by $15.93 \%$ and improve net add-in rate. Two baffling phenomena, "volume increases but revenue does not increase" and "large inflows and large outflows", can be properly mitigated in this way.

Moreover, we numerically examine how the difference between all plans always open and the dynamic plan control changes with the parameters. The result implies that initial market share is the strongest influencing factor. Mobile service providers with lower market share benefit more from the dynamic data plan control and have more potential for catch-up growth. Then we analyze the optimal policy of dynamic pricing mechanism and find plan preference in it. High-value plans are always preferred rather than low-value plans when capacity is limited.

Furthermore, customers' reservation price affects the optimal policy most among all the parameters. The reservation price reflects not only local income level but also competitors' price. If competitors' price is high, the mobile service provider 
should only open few high-value plans to maximize revenue. Otherwise, the service provider needs to open more low-value plans to compete as customers have cheap alternatives. Another parameter that significantly influences optimal policy and revenue is total alternative plan number. Mobile service providers should add new plans in total alternative plans at times instead of always opening old plans. In addition, adding high-value plans is more profitable if reservation price allows.

The remainder of this paper is organized as follows. We review relevant literature in Section 2. In Section 3, we explain models of the service provider, customers, and the stochastic dynamic programming formulation of the data plan control problem. Section 4 discusses how to optimally and efficiently solve the problem when the number of customers approaches positive infinity. In Section 5, we apply our model to a large mobile service provider in Asia. Finally, Section 6 offers some concluding remarks and future research directions.

\section{Literature Review}

In this section, we first review the literature about pricing mechanism for mobile service providers and then discuss some related work that jointly considers pricing and network congestion in Internet access.

There are mainly two pricing schemes in wireless communications: two-part tariff and three-part tariff. Under two-part tariff, a customer is charged an access price and a constant marginal price for every unit (Miravete, 2002). A reasonable combination of access and usage price can maximize a mobile service provider's revenue (Danaher, 2002). Under three-part tariff, a customer buys several allotted units at the cost of a monthly charge and pays a marginal price for units of consumption in excess of the allowance (Iyengar et al., 2007). As three-part tariff is a rational response by sophisticated service providers to consumers' misperception about their cell phone usage (Bar-Gill and Stone, 2012), it is more widely used than two-part tariff in recent years. In this paper, we analyze optimal pricing of mobile service providers 
under three-part tariff.

The design of pricing mechanism is closely related to customer behavior models. To better understand customer behavior, Lambrecht et al. (2007) investigate customer tariff choice and usage behavior under three-part tariff. They find that demand uncertainty is a key driver of choice between tariffs. When faced with both two- and three-part tariffs, customers who switched to a three-part tariff significantly "overuse" as compared with their prior two-part tariff usage because the inclusive, or "free," minutes of a three-part tariff introduces an additional attribute (Ascarza et al., 2009). In addition, customers learn rapidly to avoid an initial tendency to overconsume low-value calls, but learning is incomplete under both tariffs (Gopalakrishnan et al., 2012). Hence, by flexible and dynamic pricing, businesses could raise their marginal revenues and improve their revenue quality and profits (Tsai and Hung, 2009).

As the wireless market is competitive, some papers attempt to study why and how customers switch between carriers. Kim and Yoon (2004) point out that customer disloyalty leads to churn. This disloyalty can be due to the dissatisfaction of the provider's service or low switching cost (Lee et al., 2001; Van Ackere et al., 2013). Switching cost has been widely studied, such as Burnham et al. (2003), Pae and Hyun (2006), and Bouslah et al. (2015). To enhance and maintain customer loyalty, the fundamental essence is to ensure high customer satisfaction and develop a trustworthy relationship (Teo and Liu, 2007; Defraeye and Van Nieuwenhuyse, 2016). Fang and Zhang (2002) find that call admission control plays a significant role in providing the desired customer quality of service in wireless networks. Kim et al. (2004) suggest that call clarity and coverage are important for mobile carriers, according to customer perceptions.

Most of these above papers consider pricing of voice service and hardly any analyze pricing of data service, which becomes the core business of mobile service providers nowadays. Our paper strives to contribute to this new landscape by 
proposing a dynamic pricing mechanism of mobile data plans.

Another relevant stream of literature jointly studies price and network congestion in Internet access area. Basar and Srikant (2002) show that a service provider has the incentive to increase the available bandwidth in proportion to the number of users. Such capacity sizing is common in the Internet area but rarely seen in wireless communications due to limited wireless spectrum resource. Maglaras and Zeevi (2003) introduce a single-class Markovian model to help service providers make pricing and capacity sizing decisions. However, they set pricing mechanism from a static point of view and ignore system dynamic evolution over time. Furthermore, they assume that the congestion costs are small, which is reasonable in Internet area. However, the congestion costs are significant in wireless communication area. According to a study investigated in the UK, $45 \%$ of the negative experiences people have with their mobile can be explained by network congestion. Henderson et al. (2001) investigate congestion-dependent pricing. They assume that charging network users for the congestion they cause can lead to more efficient network utilization by forcing them to take social costs into account. However, this method also has limitations to mobile networks because of different physics mechanisms. Mobile networks present new pricing challenges.

To meet the challenges, we develop a dynamic programming model where the numbers of customers using each data plan change over time. On the basis of jointly analyzing price and customer satisfaction, this paper helps the mobile service provider maximize its revenue in long term. Table 1 compares some selective existing work with our paper according to seven dimensions. The second column tells whether the object of research is voice service or data service. The fourth column identifies whether the model is static or dynamic. The sixth column points out whether the model considers network congestion. 


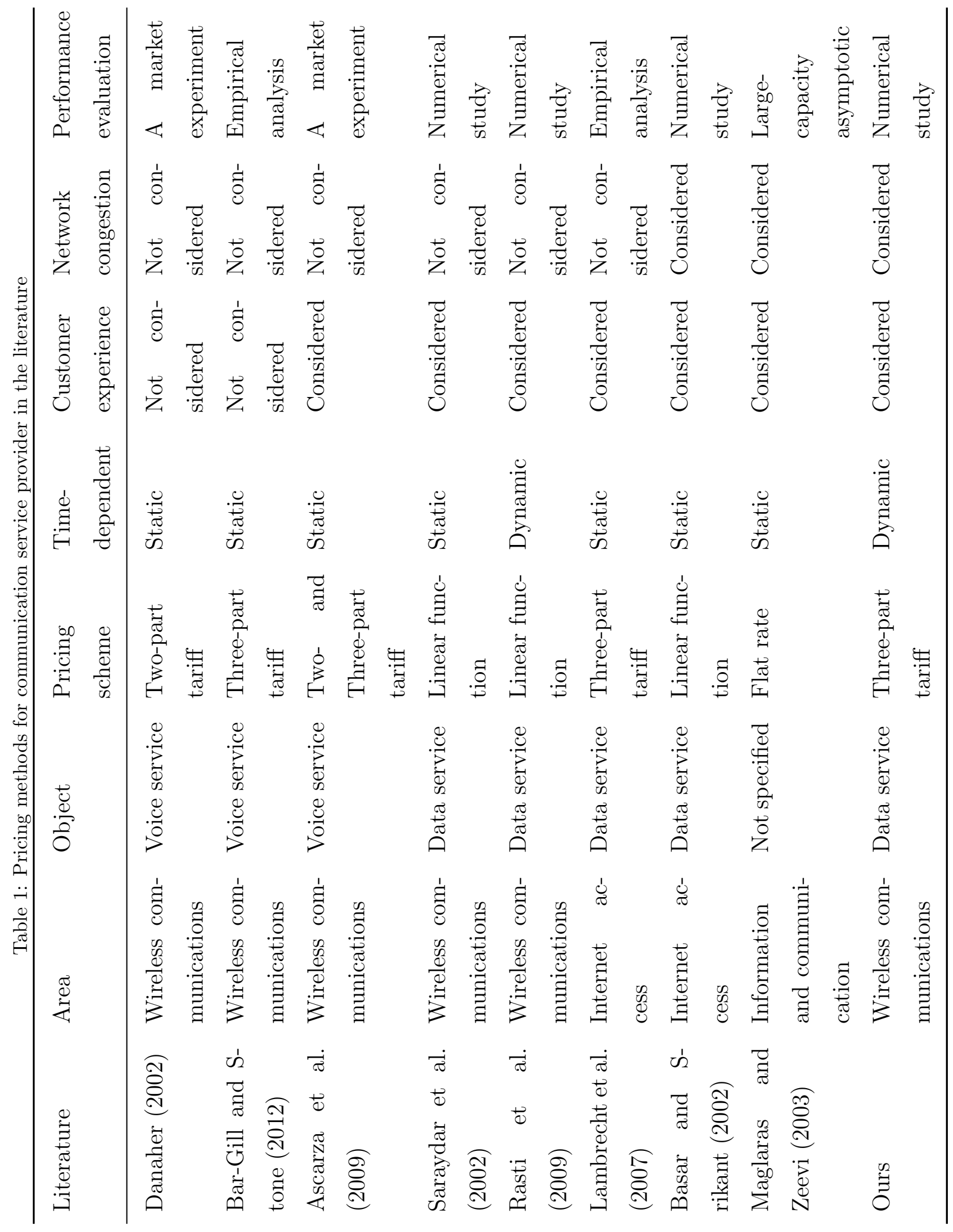




\section{Models}

\subsection{Model of the Service Provider}

We consider a market with only one mobile service provider (SP) and $N$ customers. The market has a total number of $J$ cell phone plans. Cell phone plans are based solely on data usage. Each cell phone plan $j$ has three attributes: $a_{j}$, the amount of allotted data; $b_{j}$, the monthly charge; and $c_{j}$, the overage per unit charge for data usage in excess of the allowance. On the basis of real situation, we make the following assumptions:

1. The market size is constant. As many countries in the world have reached or are approaching the complete saturation level in wireless communications, we can assume a fix total market. Once a customer leaves the SP, he enters a potential customer pool, as shown in Figure 1.

2. Congestion effects exist only in data service, not in voice and message service. As voice occupies much less bandwidth than data and has the priority to be put through first among all the wireless services, voice service does not involve congestion most of the time. For message service, as the delay cannot be observed by customers directly, we can ignore the congestion effect of text message too.

3. We focus on the control of data plans. Data service is becoming the main revenue growth engine of wireless service providers. The market of voice and message is shrinking significantly because customers instead use data service and chat software. Many wireless providers, such as AT\&T, Verizon, Sprint, and T-mobile, offer "free" voice and message and charge only data usages in "mobile share value plans." This type of plan may be the future mainstream type of plan.

4. Network capacity is proportional to the size of market. Mobile service provider$\mathrm{s}$ in different countries build different amount of base stations according to the 
market size. When the mobile service provider is located in a country with large population, it tends to provide a big network capacity (e.g. by building many base stations) to ensure customer service level and vice versa. Once the market size is determined, the network capacity of the mobile service provider is exogenously fixed.

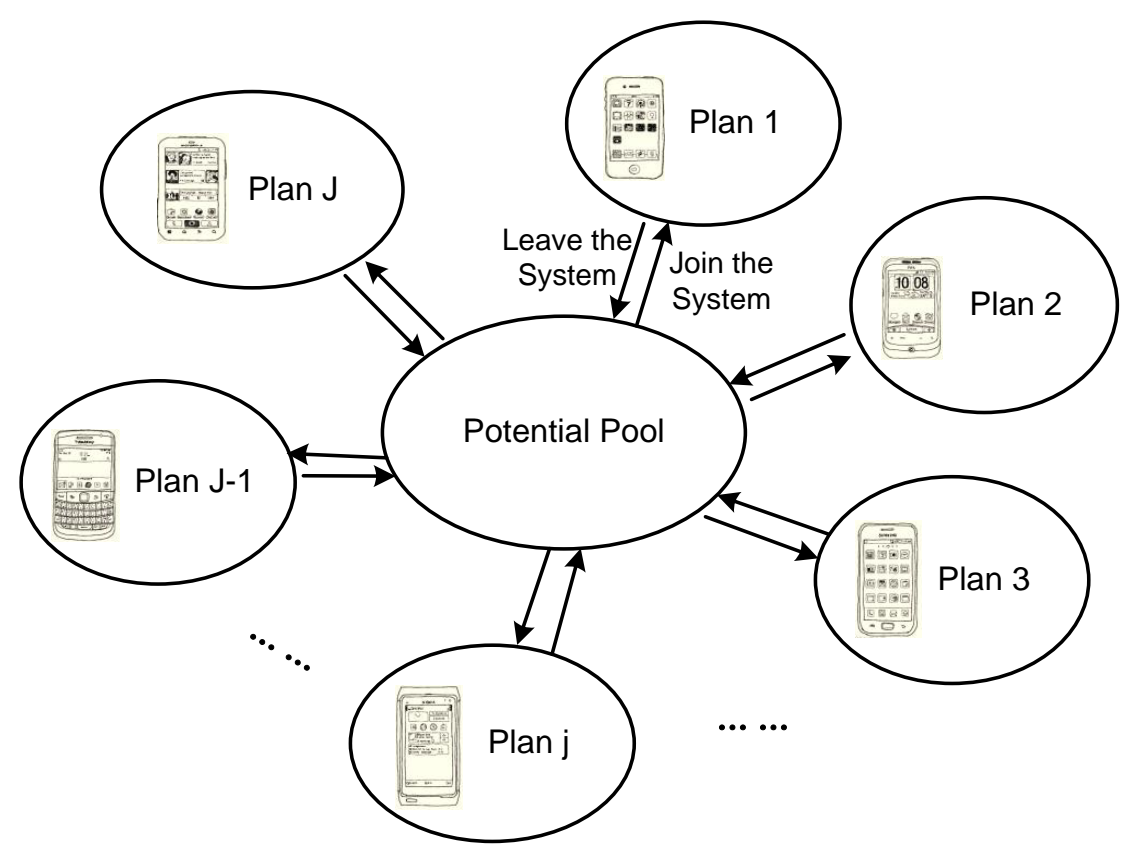

Figure 1: Constant market size and free customers flow

As the capacity is limited and congestion often occurs in data service, the SP needs to dynamically price and control mobile data plans. We dynamically open and close plans that can be seen by new customers in each period. To be specific, period $t$ consists of the following events in sequence:

1. Period $t$ begins.

2. The mobile service provider observes the numbers of customers subscribed in each cell phone plan and decides the set of plans to open.

3. Potential customers arrive and choose from the available plans.

4. All subscribed customers pay and use their plans. 
5. Because of network congestion, each subscribed customer decides whether to unsubscribe.

6. Period $t$ ends.

Figure 2 visually describes detailed flows of events and decisions in period $t$. The SP's actions and decisions are drawn in solid lines, whereas customers' actions and decisions are drawn in short dash lines. For convenience of reference, all the major notation and symbols introduced in this paper are summarized in Tables 8 and 9 .

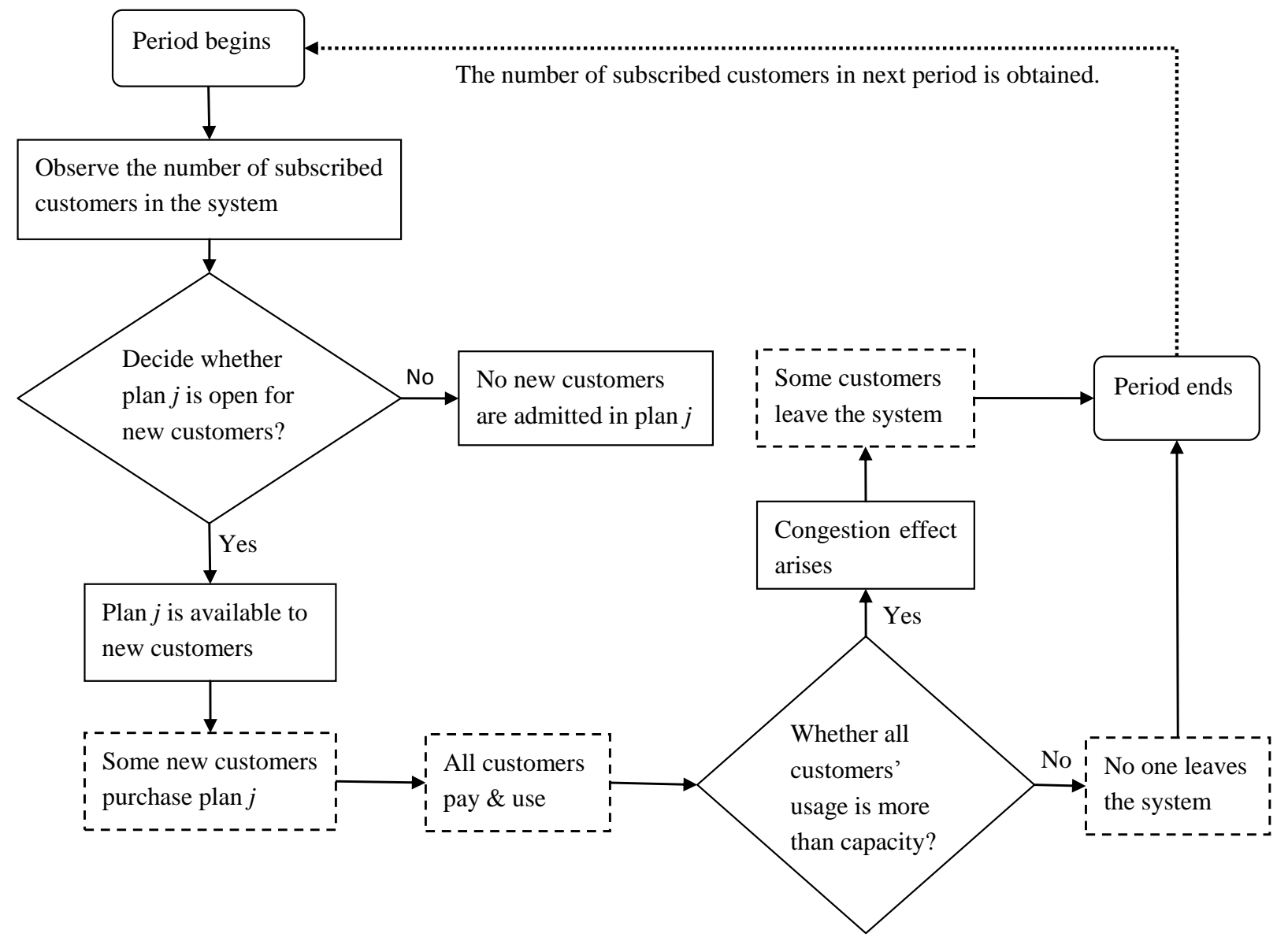

Figure 2: Flow chart of events and decisions in a single period

\subsection{Model of Customers}

We assume all potential customers are rational. In each period, a customer either subscribes to a cell phone plan or does not subscribe. We discuss how does an 
unsubscribed customer select a cell phone plan at the beginning of each period and when does a subscribed customer quit from his cell phone plan at the end of each period. Let $\tilde{D}$ be the data usage of a customer. We assume that an unsubscribed customer knows his data usage exactly (i.e., the exact realization of $\tilde{D}$ ). However, the SP knows only that a customer's data usage is drawn from the distribution of $\tilde{D}$. For convenience, we model a customer's reservation price (i.e., the cost of not subscribing any plans) as plan 0 . A customer of data usage $D$ pays $b_{0}+c_{0}\left[D-a_{0}\right]^{+}$ if he does not subscribe any plans. The constant reservation price is a special case of this form of reservation price when $a_{0}$ becomes positive infinite.

Therefore, an unsubscribed customer selects plan $j, 1 \leq j \leq J$, if it is open and

$$
b_{j}+c_{j}\left[D-a_{j}\right]^{+} \leq \min \left\{b_{0}+c_{0}\left[D-a_{0}\right]^{+}, b_{i}+c_{i}\left[D-a_{i}\right]^{+}\right\}
$$

for all plan $i$ that is open. Similarly, an unsubscribed customer does not select any cell phone plans if

$$
b_{0}+c_{0}\left[D-a_{0}\right]^{+}<b_{i}+c_{i}\left[D-a_{i}\right]^{+}
$$

for all plan $i$ that is open. Let $I_{i, t}$ equals 1 if plan $i$ is open, and 0 otherwise. For notational convenience, let $p_{j, t}$ be the probability that an unsubscribed customer selects plan $j$ to join, that is,

$$
p_{j, t}=\operatorname{Pr}\left(I_{j, t}=1, b_{j}+c_{j}\left[\tilde{D}-a_{j}\right]^{+} \leq \min _{\{0\} \cup\left\{i: I_{i, t}=1\right\}}\left\{b_{i}+c_{i}\left[\tilde{D}-a_{i}\right]^{+}\right\}\right) .
$$

Let $p_{0, t}$ be the probability that an unsubscribed customer does not select any cell phone plans, that is,

$$
p_{0, t}=\operatorname{Pr}\left(b_{0}+c_{0}\left[\tilde{D}-a_{0}\right]^{+} \leq \min _{\left\{i: I_{i, t}=1\right\}}\left\{b_{i}+c_{i}\left[\tilde{D}-a_{i}\right]^{+}\right\}\right)
$$

By definition, $\sum_{j=0}^{J} p_{j, t}=1$.

Then we proceed to the case when subscribed customers quit cell phone plans at the end of each period. Different plans' customers may have different sensitivity to network congestion. We let $\theta_{j, t}$ be the probability that a plan $j$ 's subscribed customer 
quits the service. This churn rate, $\theta_{j, t}$, is affected by many factors, an important one of which is the slow Internet connection caused by network congestion. We model $\theta_{j, t}$ as a function of system capacity and total usage, $\theta_{j, t}\left(C(N), \sum_{j=1}^{J} X_{j, t} \cdot \mathbb{E}[\tilde{D}]\right)$, where $C(N)$ is the system capacity given the total number of customers $N$ and $X_{j, t}$ is the number of plan $j$ 's subscribed customers at the beginning of period $t$. Because our solution method allows a wide range of functional forms, we will discuss the functional form in details when we discuss the solution method.

\subsection{Dynamic Programming Formulation}

We consider a discrete time multiperiod model. We use subscript $t$ to denote period $t$. A variable with subscript $t$ indicates that the variable is for period $t$. Let $\tilde{Y}_{j, t}$ be the number of customers selecting plan $j$ in period $t$. We build a multinomial model of $\tilde{\boldsymbol{Y}}_{t}$, that is,

$$
\begin{aligned}
& \operatorname{Pr}\left(\tilde{Y}_{1, t}=y_{1, t}, \tilde{Y}_{2, t}=y_{2, t}, \ldots, \tilde{Y}_{J, t}=y_{J, t}\right) \\
&= \frac{\left(N-\sum_{j=1}^{J} X_{j, t}\right) !\left(p_{1, t}\right)^{y_{1, t}}\left(p_{2, t}\right)^{y_{2, t}} \cdots\left(p_{J, t}\right)^{y_{J, t}}\left(p_{0, t}\right)^{\left(N-\sum_{j=1}^{J} X_{j, t}-\sum_{j=1}^{J} y_{j, t}\right)}}{y_{1, t} ! y_{2, t} ! \cdots y_{J, t} !\left(N-\sum_{j=1}^{J} X_{j, t}-\sum_{j=1}^{J} y_{j, t}\right) !} \\
& \forall 0 \leq \sum_{j=1}^{J} y_{j, t} \leq N-\sum_{j=1}^{J} X_{j, t} .
\end{aligned}
$$

In the above formula, $N-\sum_{j=1}^{J} X_{j, t}$ is the total number of customers in the potential pool, and $p_{j, t}$ is the probability that an unsubscribed customer selects plan $j$ in period $t$. We assume $\tilde{Y}_{j, t}$ and $\tilde{D}$ are independent in this paper. In a more general setting where the service provider would like to use $\tilde{Y}_{j, t}$ to update the distribution of $\tilde{D}$, a Bayesian analysis is needed and the model can be more complex. However, it's beyond the scope of this paper, which is to offer a modeling framework and to develop an efficient solution approach.

The number of plan $j$ 's subscribed customers at the beginning of period $t+1$, $X_{j, t+1}$, equals the number of customers subscribing plan $j$ at the beginning of period 
$t$ plus the number of customers joining plan $j$ in period $t$ minus the number of customers quitting plan $j$ in period $t$, that is, $\tilde{X}_{j, t+1}=X_{j, t}+\tilde{Y}_{j, t}-\tilde{L}_{j, t}$, where $\tilde{L}_{j, t}$ is the number of customers quitting plan $j$ in period $t$. We use a Binomial model for $\tilde{\boldsymbol{L}}_{t}$, that is, $\tilde{L}_{j, t} \sim B\left(X_{j, t}, \theta_{j, t}\right)$. The probability mass function can be expressed as

$$
\begin{aligned}
& \operatorname{Pr}\left(\tilde{\boldsymbol{L}}_{t}=\boldsymbol{l}_{t}\right) \\
= & \operatorname{Pr}\left(\tilde{L}_{1, t}=l_{1, t}\right) \cdot \operatorname{Pr}\left(\tilde{L}_{2, t}=l_{2, t}\right) \cdots \operatorname{Pr}\left(\tilde{L}_{J, t}=l_{J, t}\right) \\
= & \prod_{j=1}^{J} \frac{X_{j, t} !}{l_{j, t} !\left(X_{j, t}-l_{j, t}\right) !}\left(\theta_{j, t}\right)^{l_{j, t}}\left(1-\theta_{j, t}\right)^{X_{j, t}-l_{j, t}}, l_{j, t} \leq X_{j, t} .
\end{aligned}
$$

Here customers leaving different plans are independent events as we assume different $\tilde{L}_{j, t}{ }^{\prime} s$ are independent. However, this independence is conditional on given $\theta_{j, t}{ }^{\prime} s$, which are all increasing in the network congestion level. As a result, all $\tilde{L}_{j, t}{ }^{\prime} s$ are stochastic increasing in the network congestion level. This conditional independence assumption is merely for the ease of notation. In later limiting analysis as $N$ goes to positive infinity, because of the strong law of large number, the limiting behavior of plan $j^{\prime} s$ term $\tilde{L}_{j, t} / N$ does not depend on its correlation with another plan $\hat{j}^{\prime} s$ term $\tilde{L}_{\hat{j}, t} / N$.

We define $V_{t}\left(\boldsymbol{X}_{t}\right)$ as the mobile service provider's value function from period $t$ to the end. We consider $T$ periods. In the last period, the value function is

$$
V_{T}\left(\boldsymbol{X}_{T}\right)=\sum_{j=1}^{J} X_{j, T} \cdot \mathbb{E}\left\{b_{j}+c_{j}\left[\tilde{D}-a_{j}\right]^{+}\right\} .
$$

For any $1 \leq t<T$, the value function of period $t$ is

$$
\begin{aligned}
& V_{t}\left(\boldsymbol{X}_{t}\right) \\
= & \sum_{j=1}^{J} X_{j, t} \cdot \mathbb{E}\left\{b_{j}+c_{j}\left[\tilde{D}-a_{j}\right]^{+}\right\} \\
+ & \max _{\boldsymbol{I}_{t} \in\{0,1\}^{J}}\left\{\sum_{j=1}^{J} \mathbb{E}\left(\tilde{Y}_{j, t}\right) \cdot \mathbb{E}\left\{b_{j}+c_{j}\left[\tilde{D}-a_{j}\right]^{+}\right\}+\mathbb{E}\left[V_{t+1}\left(\tilde{\boldsymbol{X}}_{t+1}\right)\right]\right\} .
\end{aligned}
$$

The service provider's cell phone plan control problem is fully characterized by (A). In the next section, we discuss how to solve (A) optimally and efficiently. 


\section{Solution Approach}

\subsection{Limiting Analysis of Infinite $N$}

The SP's optimal cell phone plan control problem is a discrete time discrete state dynamic programming problem. The traditional approach of this type of problem is backward induction. First, we compute $V_{T}\left(\boldsymbol{X}_{T}\right)$ for all the possible $\boldsymbol{X}_{T}$. Then for any $\boldsymbol{X}_{T-1}$, we compute $V_{T-1}\left(\boldsymbol{X}_{T-1}\right)$ according to (A). Following this idea, we can compute $V_{T-2}\left(\boldsymbol{X}_{T-2}\right), V_{T-3}\left(\boldsymbol{X}_{T-3}\right), \ldots$, and $V_{1}\left(\boldsymbol{X}_{1}\right)$. A drawback of this approach is the large number of system state. This approach is efficient when $N$ is small. However, the number of system state is increasing exponentially with $N$. In real applications, $N$ is the total number of customers, which is extremely large. For this reason, it is impractical to compute $V_{t}\left(\boldsymbol{X}_{t}\right)$ for all the possible $\boldsymbol{X}_{t}$. We propose an approximation approach based on the limiting case of $N \rightarrow \infty$.

Define $\pi_{j, t}=\lim _{N \rightarrow \infty} X_{j, t} / N, \forall 1 \leq j \leq J$ and $1 \leq t \leq T$, where $\pi_{j, t}$ denotes the percentage of customers subscribed plan $j$ at the beginning of period $t$. We define $\pi_{0, t}=$ $1-\sum_{j=1}^{J} \pi_{j, t}$. Thus the system state can be described as $\boldsymbol{\pi}_{t}=\left(\pi_{0, t}, \pi_{1, t}, \ldots, \pi_{J, t}\right)$. By definition, the sum of all elements of $\hat{\boldsymbol{\pi}}_{t}$ equals 1. Define $\bar{V}_{t}\left(\boldsymbol{\pi}_{t}\right)$ as the average revenue per person when the customer population approaches infinity. For the last period $T$, we define

$$
\bar{V}_{T}\left(\boldsymbol{\pi}_{T}\right)=\sum_{j=1}^{J} \pi_{j, T} \cdot \mathbb{E}\left\{b_{j}+c_{j}\left[\tilde{D}-a_{j}\right]^{+}\right\} .
$$

In the previous discussion, we model the churn rate $\theta_{j, t}$ as a function of system capacity $C(N)$ and total usage $\sum_{j=1}^{J} X_{j, t} \cdot \mathbb{E}[\tilde{D}]$. We assume the following limit

exists, $\lim _{N \rightarrow+\infty} \theta_{j, t}\left(C(N), \sum_{j=1}^{J} X_{j, t} \cdot \mathbb{E}[\tilde{D}]\right)$. The limit simply argues that when the system is infinitely large, the equilibrium churn rate exists. Furthermore, we assume that this limit can be described by the following functional form:

$$
\begin{aligned}
& \lim _{N \rightarrow+\infty} \theta_{j, t}\left(C(N), \sum_{j=1}^{J} X_{j, t} \cdot E[\tilde{D}]\right) \\
= & \theta_{j, t}\left(\lim _{N \rightarrow+\infty} \frac{X_{1, t}}{N}, \lim _{N \rightarrow+\infty} \frac{X_{2, t}}{N}, \ldots, \lim _{N \rightarrow+\infty} \frac{X_{J, t}}{N}\right)=\theta_{j, t}\left(\boldsymbol{\pi}_{t}\right) .
\end{aligned}
$$


The function of $\theta_{j, t}\left(\boldsymbol{\pi}_{t}\right)$ can take any general forms. For illustrative purposes, we assume $\theta_{j, t}\left(\boldsymbol{\pi}_{t}\right)$ equals $\bar{\theta}_{j}$ if $\sum_{j=1}^{J} \alpha_{j} \pi_{j, t} \geq \Omega$, and 0 otherwise in the following discussion. Here, $\Omega$ is the threshold of network usage above which the network is congested.

For any $1 \leq t<T$, we define

$$
\begin{aligned}
& \bar{V}_{t}\left(\boldsymbol{\pi}_{t}\right) \\
= & \sum_{j=1}^{J} \pi_{j, t} \cdot \mathbb{E}\left\{b_{j}+c_{j}\left[\tilde{D}-a_{j}\right]^{+}\right\} \\
+ & \max _{\boldsymbol{I}_{t} \in\{0,1\}^{J}}\left\{\left(1-\sum_{j=1}^{J} \pi_{j, t}\right) \cdot \sum_{j=1}^{J}\left(p_{j, t} \cdot \mathbb{E}\left\{b_{j}+c_{j}\left[\tilde{D}-a_{j}\right]^{+}\right\}\right)+\bar{V}_{t+1}\left(\boldsymbol{A}_{t}\left(\boldsymbol{I}_{t}, \boldsymbol{\pi}_{t}\right) \cdot \boldsymbol{\pi}_{t}\right)\right\},
\end{aligned}
$$

where $\boldsymbol{A}_{t}$ is a $(J+1) \times(J+1)$ matrix and its matrix form is:

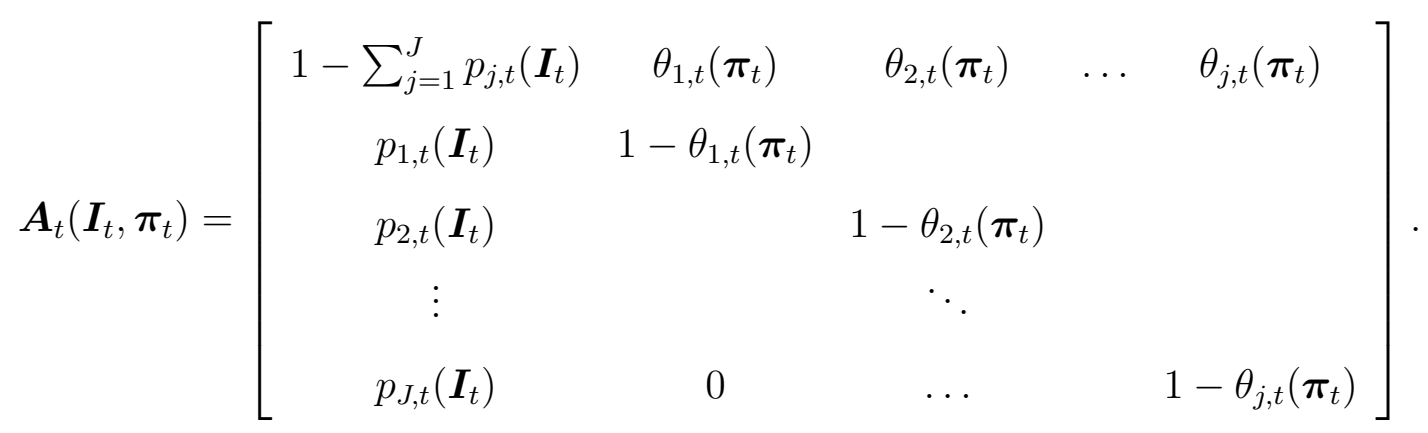

$\boldsymbol{A}_{t}\left(\boldsymbol{I}_{t}, \boldsymbol{\pi}_{t}\right)$ is a function of $\boldsymbol{I}_{t}$ and $\boldsymbol{\pi}_{t}$ because $p_{j, t}$ is a function of $\boldsymbol{I}_{t}$, and $\theta_{j, t}$ depends on $\boldsymbol{\pi}_{t}$.

\section{Proposition 1.}

$$
\lim _{N \rightarrow \infty} \frac{V_{t}\left(\boldsymbol{X}_{t}\right)}{N}=\bar{V}_{t}\left(\boldsymbol{\pi}_{t}\right), \forall 1 \leq t \leq T
$$

Proposition 1 suggests that when $N$ is sufficiently large, we can equivalently solve a dynamic programming problem with continuous states. In the following subsection, we show that $\bar{V}_{t}\left(\boldsymbol{\pi}_{t}\right)$ is easier to compute than $V_{t}\left(\boldsymbol{X}_{t}\right)$.

\subsection{Integer linear programming Formulation}

We provide an equivalent integer linear programming formulation to compute $\bar{V}_{t}\left(\boldsymbol{\pi}_{t}\right)$. The main nonlinearity of the control problem is the system state transition equation $\boldsymbol{\pi}_{t+1}=\boldsymbol{A}_{t}\left(\boldsymbol{I}_{t}, \boldsymbol{\pi}_{t}\right) \cdot \boldsymbol{\pi}_{t}$, where both $\boldsymbol{A}_{t}\left(\boldsymbol{I}_{t}, \boldsymbol{\pi}_{t}\right)$ and $\boldsymbol{\pi}_{t}$ involve decision variables. 
Define $S\left(\boldsymbol{I}_{t}, 0\right)=\boldsymbol{A}\left(\boldsymbol{I}_{t}, \boldsymbol{\pi}_{t}\right)$ for any $\boldsymbol{\pi}_{t}$ that satisfies $\sum_{j=1}^{J} \alpha_{j} \pi_{j, t}<\Omega$ and define $S\left(\boldsymbol{I}_{t}, 1\right)=\boldsymbol{A}\left(\boldsymbol{I}_{t}, \boldsymbol{\pi}_{t}\right)$ for any $\boldsymbol{\pi}_{t}$ that satisfies $\sum_{j=1}^{J} \alpha_{j} \pi_{j, t} \geq \Omega$. For any $\boldsymbol{I}_{t}$, both $S\left(\boldsymbol{I}_{t}, 1\right)$ and $S\left(\boldsymbol{I}_{t}, 0\right)$ can be computed and treated as given parameters. The system state transition becomes $\boldsymbol{\pi}_{t+1}=S\left(\boldsymbol{I}_{t}, 1\right) \cdot \boldsymbol{\pi}_{t}$ if the plan control is $\boldsymbol{I}_{t}$ and the network is congested, and $\boldsymbol{\pi}_{t+1}=S\left(\boldsymbol{I}_{t}, 0\right) \cdot \boldsymbol{\pi}_{t}$ if the plan control is $\boldsymbol{I}_{t}$ and the network is not congested. With the definition of $S\left(\boldsymbol{I}_{t}, 1\right)$ and $S\left(\boldsymbol{I}_{t}, 0\right)$, we are able to formulate the system state transition equation as a mixed integer linear function.

Introduce binary decision variables $K_{\boldsymbol{I}_{t}, t} \in\{0,1\}$ for each $\boldsymbol{I}_{t} . K_{\boldsymbol{I}_{t}, t}$ equals 1 if control $\boldsymbol{I}_{t}$ is used. By definition, we have

$$
\sum_{\boldsymbol{I}_{t} \in\{0,1\}^{J}} K_{\boldsymbol{I}_{t}, t}=1,
$$

that is, only one control can be used in each period. Further, we introduce a binary decision variable $L_{t} \in\{1,0\}$ to denote whether the network is congested. $L_{t}$ equals 1 if $\sum_{j=1}^{J} \alpha_{j} \pi_{j, t} \geq \Omega$ (i.e., congestion effects arise), and 0 otherwise. This logic can be achieved by using a very large number $M$ :

$$
-M \cdot\left(1-L_{t}\right) \leq \sum_{j=1} \alpha_{j} \cdot \pi_{j, t}-\Omega \leq M \cdot L_{t}
$$

The system state transition equation can be then formulated as mixed integer linear equations:

$$
\begin{aligned}
& \boldsymbol{\pi}_{t+1} \geq S\left(\boldsymbol{I}_{t}, 1\right) \cdot \boldsymbol{\pi}_{t}-M \cdot\left(1-K_{\boldsymbol{I}_{t}, t}\right)-M \cdot\left(1-L_{t}\right), \forall 1 \leq t<T, \boldsymbol{I}_{t} \in\{0,1\}^{J}, \\
& \boldsymbol{\pi}_{t+1} \geq S\left(\boldsymbol{I}_{t}, 0\right) \cdot \boldsymbol{\pi}_{t}-M \cdot\left(1-K_{\boldsymbol{I}_{t}, t}\right)-M \cdot L_{t}, \forall 1 \leq t<T, \boldsymbol{I}_{t} \in\{0,1\}^{J}, \\
& \sum_{j=0}^{J} \pi_{j, t}=1, \forall 1 \leq t \leq T .
\end{aligned}
$$

Another nonlinear term is

$$
\begin{aligned}
& \left(1-\sum_{j=1}^{J} \pi_{j, t}\right) \cdot \sum_{j=1}^{J} p_{j, t} \cdot \mathbb{E}\left[b_{j}+c_{j}\left[\tilde{D}-a_{j}\right]^{+}\right] \\
= & \pi_{0, t} \cdot \sum_{j=1}^{J} p_{j, t} \cdot \mathbb{E}\left[b_{j}+c_{j}\left[\tilde{D}-a_{j}\right]^{+}\right] \\
= & \pi_{0, t} \cdot \sum_{j=1}^{J} \sum_{\boldsymbol{I}_{t} \in\{0,1\}^{J}} p_{j, t}\left(\boldsymbol{I}_{t}\right) \cdot K_{\boldsymbol{I}_{t}, t} \cdot \mathbb{E}\left[b_{j}+c_{j}\left[\tilde{D}-a_{j}\right]^{+}\right] .
\end{aligned}
$$


The term $\pi_{0, t} \cdot K_{\boldsymbol{I}_{t}, t}$ is nonlinear. We introduce $\Pi_{\boldsymbol{I}_{t}, t}$ and add $\Pi_{\boldsymbol{I}_{t}, t} \leq \pi_{0, t}, \Pi_{\boldsymbol{I}_{t}, t} \leq K_{\boldsymbol{I}_{t}, t}$. A revenue maximization problem would guarantee that $\Pi_{\boldsymbol{I}_{t}, t}=\pi_{0, t} \cdot K_{\boldsymbol{I}_{t}, t}$ is satisfied at the optimal solution. Then, we have

$$
\begin{aligned}
& \Pi_{\boldsymbol{I}_{t}, t} \leq \pi_{0, t}, \forall \boldsymbol{I}_{t} \in\{0,1\}^{J}, 1 \leq t<T, \\
& \Pi_{\boldsymbol{I}_{t}, t} \leq K_{\boldsymbol{I}_{t}, t}, \forall \boldsymbol{I}_{t} \in\{0,1\}^{J}, 1 \leq t<T .
\end{aligned}
$$

Let $z_{p}(\overline{\boldsymbol{\pi}})$ be the optimal value of the SP's optimization problem when the initial state is $\overline{\boldsymbol{\pi}}$.

$$
\begin{aligned}
z_{p}(\overline{\boldsymbol{\pi}})=\max & \sum_{t=1}^{T} \sum_{j=1}^{J} \mathbb{E}\left\{b_{j}+c_{j}\left[\tilde{D}-a_{j}\right]^{+}\right\} \cdot \pi_{j, t}+ \\
& \sum_{t=1}^{T-1} \sum_{\boldsymbol{I}_{t} \in\{0,1\}^{J}}\left(\sum_{j=1}^{J} p_{j, t}\left(\boldsymbol{I}_{t}\right) \cdot \mathbb{E}\left\{b_{j}+c_{j}\left[\tilde{D}-a_{j}\right]^{+}\right\}\right) \cdot \Pi_{\boldsymbol{I}_{t}, t} \\
\text { s.t. } & (T R),(C T),(C G),(P K) \\
& \pi_{j, 1}=\bar{\pi}_{j}, \forall 0 \leq j \leq J, \\
& K_{\boldsymbol{I}_{t}, t} \in\{0,1\}, \forall \boldsymbol{I}_{t} \in\{0,1\}^{J}, 1 \leq t<T \\
& L_{t} \in\{0,1\}, \forall 1 \leq t<T, \\
& \pi_{j, t} \geq 0, \forall 0 \leq j \leq J, 1 \leq t \leq T, \\
& \Pi_{\boldsymbol{I}_{t}, t} \geq 0, \forall \boldsymbol{I}_{t} \in\{0,1\}^{J}, 1 \leq t<T .
\end{aligned}
$$

\section{Proposition 2.}

$$
z_{p}(\overline{\boldsymbol{\pi}})=\bar{V}_{1}(\overline{\boldsymbol{\pi}})
$$

Proposition 2 indicates that this integer linear programming formulation is equivalent to previously proposed dynamic programming formulation. We can solve the dynamic control problem through mixed integer linear programming. However, the number of variables is still very large. Approximately, the number of variables is proportional to $O\left(2^{J} * T\right)$. In the next subsection, we work to reduce the size of the integer linear programming problem. 


\subsection{Dimension Reduction}

The high dimensionality is mainly caused by $p_{j, t}\left(\boldsymbol{I}_{t}\right)$. There are $2^{J}$ possible $\boldsymbol{I}_{t}$. We consider another approach to determine $p_{j, t}$.

Assumption 1. For the plan attributes $a, b$ and $c$, we have

1. $a_{j+1}>a_{j}+\left(b_{j+1}-b_{j}\right) / c_{j}, \forall 1 \leq j<J$,

2. $b_{1}<b_{2}<\ldots<b_{J}$,

3. $c_{1} \geq c_{2} \geq \ldots \geq c_{J}$.

Assumption 1 illustrates high-value plans have more allotted data and lower overage per unit charge. As illustrated in Figure 3 , for any plan $j$, if $1 \leq i_{1}<j<$ $i_{2} \leq J$, let $X_{i_{1}, j}$ be the data usage below which plan $i_{1}$ is preferred over plan $j$ and let $X_{j, i_{2}}$ be the data usage above which plan $i_{2}$ is preferred over plan $j$. We model customers' reservation price as plan 0 . From figure 3 , we can find that $p_{j, t}$ can be determined by following constraints:

$p_{j, t} \leq M \cdot I_{j, t}, \forall 1 \leq t<T, 1 \leq j \leq J$

$p_{j, t} \leq\left[F\left(X_{j, i_{2}}\right)-F\left(X_{i_{1}, j}\right)\right]+M\left(2-I_{i_{1}, t}-I_{i_{2}, t}\right), \forall 1 \leq i_{1}<j<i_{2} \leq J$, (plans $\left.i_{1}, j, i_{2}\right)$

$p_{j, t} \leq\left[F\left(X_{j, i_{2}}\right)-F\left(X_{0, j}\right)\right]+M\left(1-I_{i_{2}, t}\right), \forall 1 \leq j<i_{2} \leq J$, (reservation plan, plans $\left.j, i_{2}\right)$

$p_{j, t} \leq\left[1-F\left(X_{i 1, j}\right)\right]+M\left(1-I_{i_{1}, t}\right), \forall 1 \leq i_{1}<j \leq J,\left(\right.$ plans $\left.i_{1}, j\right)$

$p_{j, t} \leq\left[1-F\left(X_{0, j}\right)\right], \forall 1 \leq j \leq J,(\operatorname{plan} j)$

$p_{0, t} \leq F\left(X_{0, i_{2}}\right)+M\left(1-I_{i 2, t}\right), \forall 1 \leq i_{2} \leq J$, (reservation plan, plan $\left.i_{2}\right)$

$\sum_{j=0}^{J} p_{j, t}=1, \forall 1 \leq t<T$,

where $F(X)$ is the c.d.f of the random variable $\tilde{D}$.

Let $\beta_{j, t}$ be the percentage of customers joining plan $j$ from potential pool in period $t$ and $\gamma_{j, t}$ be the percentage of customers leaving the system from plan $j$ in 


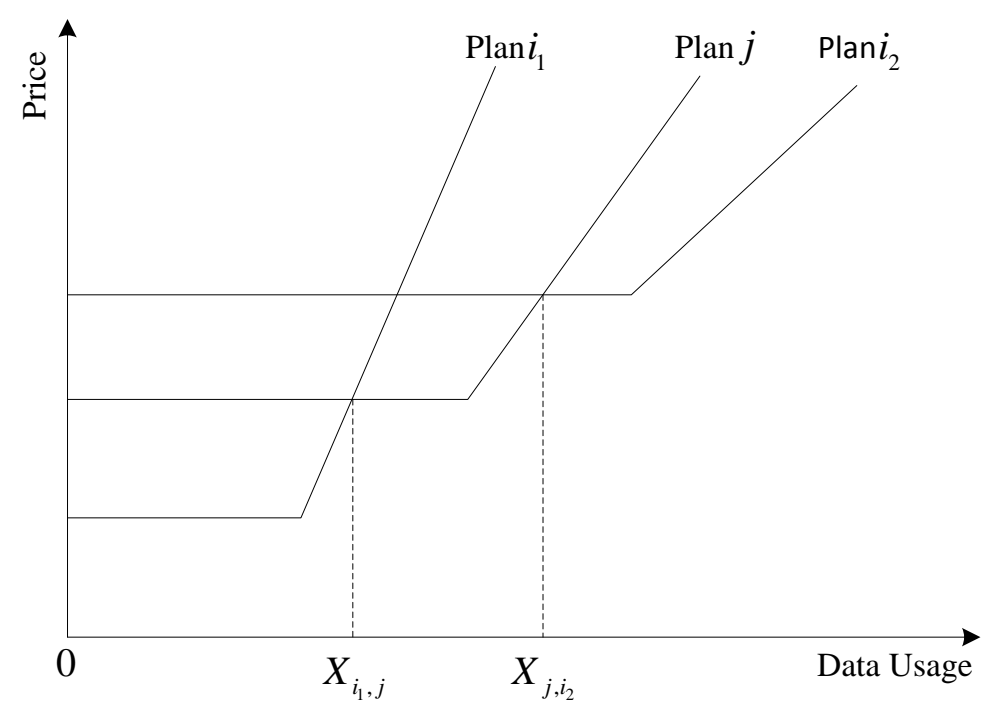

Figure 3: Different plans' prices with respect to data usages

period $t$. Hence, $\beta_{j, t}$ can be determined by:

$$
\begin{aligned}
& \beta_{j, t} \leq M \cdot I_{j, t}, \forall 1 \leq t<T, 1 \leq j \leq J, \\
& \beta_{j, t} \leq \pi_{0, t} \cdot p_{j, t}+M\left(1-I_{j, t}\right), \forall 1 \leq t<T, 1 \leq j \leq J, \\
& \beta_{j, t} \geq-M \cdot I_{j, t}, \forall 1 \leq t<T, 1 \leq j \leq J, \\
& \beta_{j, t} \geq \pi_{0, t} \cdot p_{j, t}-M\left(1-I_{j, t}\right), \forall 1 \leq t<T, 1 \leq j \leq J .
\end{aligned}
$$

Because $\pi_{0, t} \cdot p_{j, t}$ is a nonlinear expression, we consider to introduce a new variable $p p_{j, t}$ to replace $\pi_{0, t} \cdot p_{j, t}$. We define

$$
p p_{j, t}=\pi_{0, t} \cdot p_{j, t}
$$


Then we add the following constraints:

$$
\begin{aligned}
& p p_{j, t} \leq M \cdot I_{j, t}, \forall 1 \leq t<T, 1 \leq j \leq J \\
& p p_{j, t} \leq \pi_{0, t} \cdot\left[F\left(X_{j, i_{2}}\right)-F\left(X_{i_{1}, j}\right)\right]+M\left(2-I_{i_{1}, t}-I_{i_{2}, t}\right), \forall 1 \leq i_{1}<j<i_{2} \leq J \\
& p p_{j, t} \leq \pi_{0, t} \cdot\left[F\left(X_{j, i_{2}}\right)-F\left(X_{0, j}\right)\right]+M\left(1-I_{i_{2}, t}\right), \forall 1 \leq j<i_{2} \leq J \\
& p p_{j, t} \leq \pi_{0, t} \cdot\left[1-F\left(X_{i_{1}, j}\right)\right]+M\left(1-I_{i_{1}, t}\right), \forall 1 \leq i_{1}<j \leq J \\
& p p_{j, t} \leq \pi_{0, t} \cdot\left[1-F\left(X_{0, j}\right)\right], \forall 1 \leq j \leq J \\
& p p_{0 . t} \leq \pi_{0, t} \cdot F\left(X_{0, i_{2}}\right)+M \cdot\left(1-I_{i_{2}, t}\right), \forall 1 \leq i_{2} \leq J \\
& \sum_{j=0}^{J} p p_{j, t}=\pi_{0, t}, \forall 1 \leq t<T
\end{aligned}
$$

The equation (JP) turns into

$$
\begin{aligned}
& \beta_{j, t} \leq M \cdot I_{j, t}, \forall 1 \leq t<T, 1 \leq j \leq J \\
& \beta_{j, t} \leq p p_{j, t}+M\left(1-I_{j, t}\right), \forall 1 \leq t<T, 1 \leq j \leq J \\
& \beta_{j, t} \geq-M \cdot I_{j, t}, \forall 1 \leq t<T, 1 \leq j \leq J \\
& \beta_{j, t} \geq p p_{j, t}-M\left(1-I_{j, t}\right), \forall 1 \leq t<T, 1 \leq j \leq J .
\end{aligned}
$$

On the basis of the above analysis, we consider the following integer linear program- 
ming problem.

$$
\begin{aligned}
\hat{z}_{p}(\overline{\boldsymbol{\pi}})=\max & \sum_{t=1}^{T} \sum_{j=1}^{J} \mathbb{E}\left\{b_{j}+C_{j}\left[\tilde{D}-a_{j}\right]^{+}\right\} \cdot \pi_{j, t}+\sum_{t=1}^{T-1} \sum_{j=1}^{J} \beta_{j, t} \cdot \mathbb{E}\left\{b_{j}+C_{j}\left[\tilde{D}-a_{j}\right]^{+}\right\} \\
\text {s.t. } & (P P),\left(J P^{\prime}\right),(C G), \\
& \pi_{0, t+1}=\pi_{0, t}-\sum_{j=1}^{J} \beta_{j, t}+\sum_{j=1}^{J} \gamma_{j, t}, \forall 1 \leq t<T \\
& \pi_{j, t+1}=\pi_{j, t}+\beta_{j, t}-\gamma_{j, t}, \forall 1 \leq t<T, 1 \leq j \leq J \\
& \gamma_{j, t} \geq \pi_{j, t} \bar{\theta}_{j}-M\left(1-L_{t}\right), \forall 1 \leq t<T, 1 \leq j \leq J \\
& \gamma_{j, t} \geq-M \cdot L_{t}, \forall 1 \leq t<T, 1 \leq j \leq J \\
& \gamma_{j, t} \leq \pi_{j, t} \bar{\theta}_{j}+M\left(1-L_{t}\right), \forall 1 \leq t<T, 1 \leq j \leq J \\
& \gamma_{j, t} \leq M \cdot L_{t}, \forall 1 \leq t<T, 1 \leq j \leq J \\
J & \sum_{j=0} \pi_{j, t}=1, \\
& \pi_{j, t} \geq 0, \forall 1 \leq t \leq T, 1 \leq j \leq J \\
& I_{j, t} \in(0,1), \forall 1 \leq t<T, 1 \leq j \leq J \\
& L_{t} \in(0,1), \forall 1 \leq t<T . \\
&
\end{aligned}
$$

\section{Proposition 3.}

$$
\hat{z}_{p}(\overline{\boldsymbol{\pi}})=z_{p}(\overline{\boldsymbol{\pi}})=\bar{V}_{1}(\overline{\boldsymbol{\pi}})
$$

Proposition 3 suggests that the integer linear programming problem's dimension can be significantly reduced to $O(J * T)$. We infer that in practice, reasonably sized problems can be solved efficiently.

\section{Numerical Application}

In this section, we apply our model to a large mobile service provider in Asia. In particular, we aim to solve the problem of "volume increases but revenue does not increase" and "large inflows and large outflows" phenomena, which also widely exist in many other mobile service providers around the world. 


\subsection{Data and Parameters}

This large mobile service provider has provided us with 10,000 customers' data usages from March 2012 to February 2013. During this period, the SP provided over 30 cell phone plans in three categories (A, B, and $\mathrm{C}$ ) to customers. In this numerical application, we focus on five plans in category A with 1,500 customers. The common feature of these five plans in category $\mathrm{A}$ is that they have no message allowance and few voice allowance. In other words, they resemble the most the data plans considered in this paper.

These five plans' monthly charge $b_{j}$, amount of allotted data $a_{j}$ and overage per unit charge $c_{j}$ can be found on the SP's website. Figure 4 shows the relationship between data usage (in MB) and price (in RMB) of these five plans. In Table 2, we assume each plan's percentage of contribution to cause congestion $\alpha_{j}$, customers churn rate $\theta_{j}$ based on the real data. Distribution fit of 1,500 customers' data usages suggests that customer's data usage can be fitted as $\operatorname{Exp}(0.00418) \mathrm{MB}$.

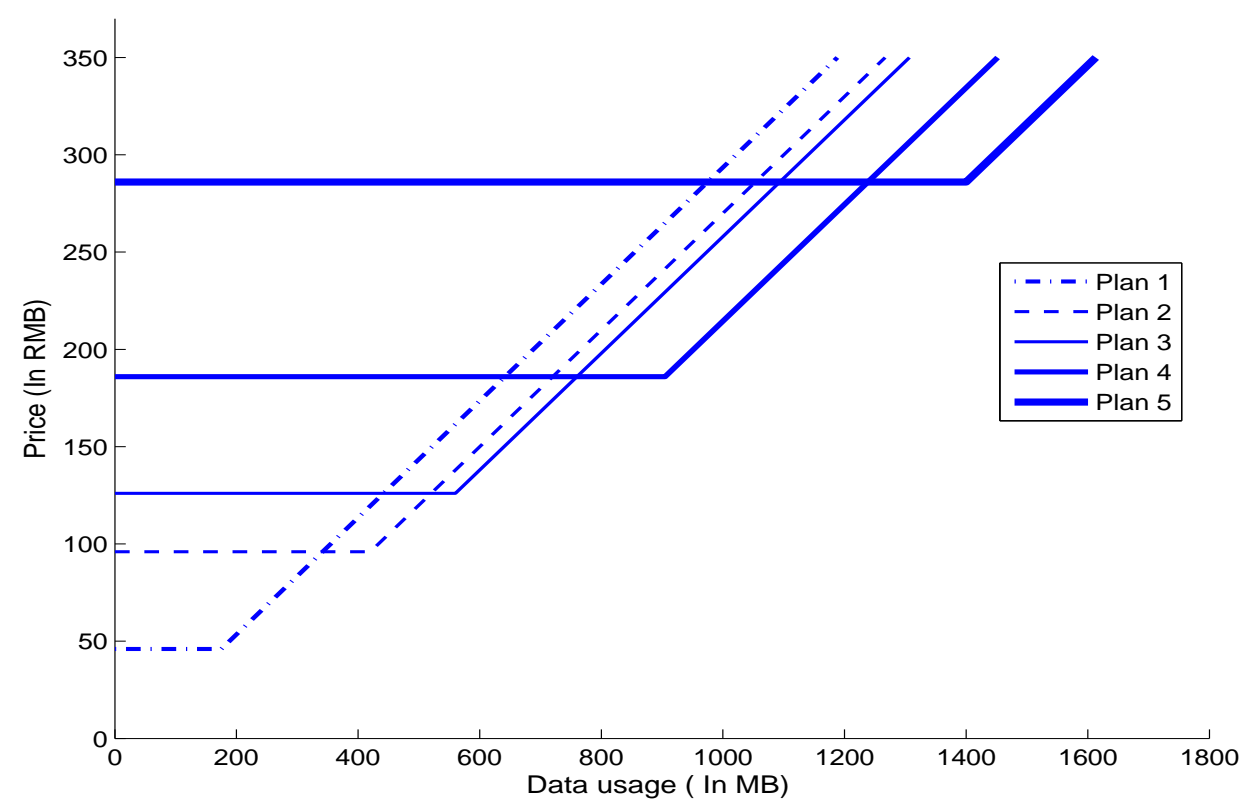

Figure 4: Five plans' prices with respect to data usages

In this numerical study, we consider that the total period is 5 . The threshold of customers' data usage $\Omega$ is assumed to be 0.85 . To evaluate the effect of our 
Table 2: Characters of Chosen 5 Plans

\begin{tabular}{llllll} 
Plan ID: $j$ & $a_{j}$ & $b_{j}$ & $c_{j}$ & $\alpha_{j}$ & $\theta_{j}$ \\
\hline 1 & 175 & 46 & 0.3 & 0.06 & 0.003 \\
2 & 420 & 96 & 0.3 & 0.12 & 0.006 \\
3 & 560 & 126 & 0.3 & 0.16 & 0.008 \\
4 & 905 & 186 & 0.3 & 0.26 & 0.013 \\
5 & 1400 & 286 & 0.3 & 0.40 & 0.020 \\
\hline
\end{tabular}

method, we compare the proposed dynamic plan control with the strategy of always opening all plans and examine how their difference changes with parameters.

Further, we numerically analyze the preference between any two plans and test different parameters' impact on the optimal policy under dynamic pricing mechanism. Consequently, practical implications are also discussed.

\subsection{Results and Implications}

After comparing dynamic plan control and all plans always open strategy, Table 3 reports the numerical results. The dynamic plan control policy increases the total revenue by $15.93 \%$. The "volume increases but revenue does not increase" phenomenon is properly mitigated. At the same time, dynamic pricing improves the net add-in rate. We find that the net add-in rate is tremendous high in the first period but drops to very low or even negative in periods $2-4$ when all plans are always open. By contrast, dynamic pricing mechanism has a longer-term horizon and pays attention to the balance of network capacity and total data usages in the network. The "large inflows and large outflows" phenomenon is improved by dynamic plan control.

When we examine how the difference between all plans always open and dynamic plan control changes with the parameters, $\bar{\pi}_{j}$, the initial percentage of customers subscribed plan $j$, is found as the strongest influencing factor. As shown in Table 
Table 3: Comparison between all plans always open and dynamic plan control

\begin{tabular}{|c|c|c|c|c|}
\hline \multirow[b]{2}{*}{$t$} & \multicolumn{2}{|c|}{ All Plans Always Open } & \multicolumn{2}{|c|}{ Dynamic Plan Control } \\
\hline & Net Add-in Rate & Total Revenue & New Add-in Rate & Total Revenue \\
\hline 1 & 0.32949083 & & 0.14297541 & \\
\hline 2 & -0.071459075 & & 0.015649718 & \\
\hline 3 & 0.005279336 & 544.095 & 0.103288354 & 630.767 \\
\hline 4 & 0.0003082 & & 0.00795785 & \\
\hline 5 & - & & - & \\
\hline
\end{tabular}

4 , low $\bar{\pi}_{j}$ group has the lowest revenue 496.866 under all plans always open strategy and has the highest revenue 669.151 under dynamic plan control strategy. Dynamic plan control helps low $\bar{\pi}_{j}$ group increase revenue by $34.67 \%$. It's easy to find that the revenue difference of two strategies increases while $\bar{\pi}_{j}$ decreases. This result implies that a mobile service provider whose initial market share is low can benefit more from the dynamic data plan control.

In different parameter settings, we analyze the optimal policy of dynamic pricing mechanism and find plan preference in it. Except high $\Omega$ group, high-value plans are always preferred rather than low-value plans as shown in Table 5. That is, if a low-value plan is open in the optimal policy, plans higher than it must be open simultaneously when capacity is not enough. However, when $\Omega$ is high and capacity is large enough, the preference does not exist. For example, plan 4 is open while plan 5 is closed in period 1 of high $\Omega$ group. In real life, mobile service provider's capacity is fixed and limited in most cases. Hence, it can help the SP simplify dynamic plan control process based on the plan preference.

Moreover, customers' reservation price, $R$, affects the optimal policy most among all the parameters. Table 6 shows the three groups of reservation prices being tested. For instance, in the low reservation price group, a customer with data usage $\tilde{D} \in[0,200]$ has a reservation price 50. As Figure 5, the SP tends to open more plans and more low-value plans when customers' reservation price is low. However, 
Table 4: The influence of $\bar{\pi}_{j}$ to the difference of two methods

\begin{tabular}{lllll}
\hline & & Low $\bar{\pi}_{j}$ & Medium $\bar{\pi}_{j}$ & High $\bar{\pi}_{j}$ \\
\hline Settings & $\bar{\pi}_{1}$ & 0.05 & 0.1 & 0.15 \\
& $\bar{\pi}_{2}$ & 0.1 & 0.19 & 0.28 \\
& $\bar{\pi}_{3}$ & 0.04 & 0.09 & 0.13 \\
& $\bar{\pi}_{4}$ & 0.03 & 0.06 & 0.09 \\
& $\bar{\pi}_{5}$ & 0.007 & 0.014 & 0.02 \\
\hline \multirow{2}{*}{ Results } & Revenue of all plans al- & 496.866 & 522.083 & 544.095 \\
& ways open & & & \\
& Revenue of dynamic & 669.151 & 650.072 & 630.767 \\
& plan control & & & \\
& Revenue difference & 172.285 & 127.989 & 86.672 \\
& Percentage of revenue & $34.67 \%$ & $24.52 \%$ & $15.93 \%$ \\
& increment & & & \\
\hline
\end{tabular}

when customers' reservation price becomes high, the SP has the motivation to close low-value plans and only open a small amount of high-value plans.

In practical implication, mobile service providers in countries of different development levels should take different pricing strategies as people's reservation price is different. In developed countries, a SP should only open a small amount of highvalue plans. However, in developing countries, a SP should open more and low-value plans. Even in a same country, the SP needs open different plans in districts of different income level.

From another perspective, reservation price is also strongly correlated with competitors' price. If competitors' price is high, the SP should only open few high-value plans to maximize revenue because customers have no way but buy high-value plans in this situation. However, if the competitors' price is low, the SP needs to open more and low-value plans to compete as customers have cheap alternatives.

Another parameter that significantly influences the SP's optimal policy and revenue is total alternative plan number $J$. Table 7 shows the SP's revenue increases 


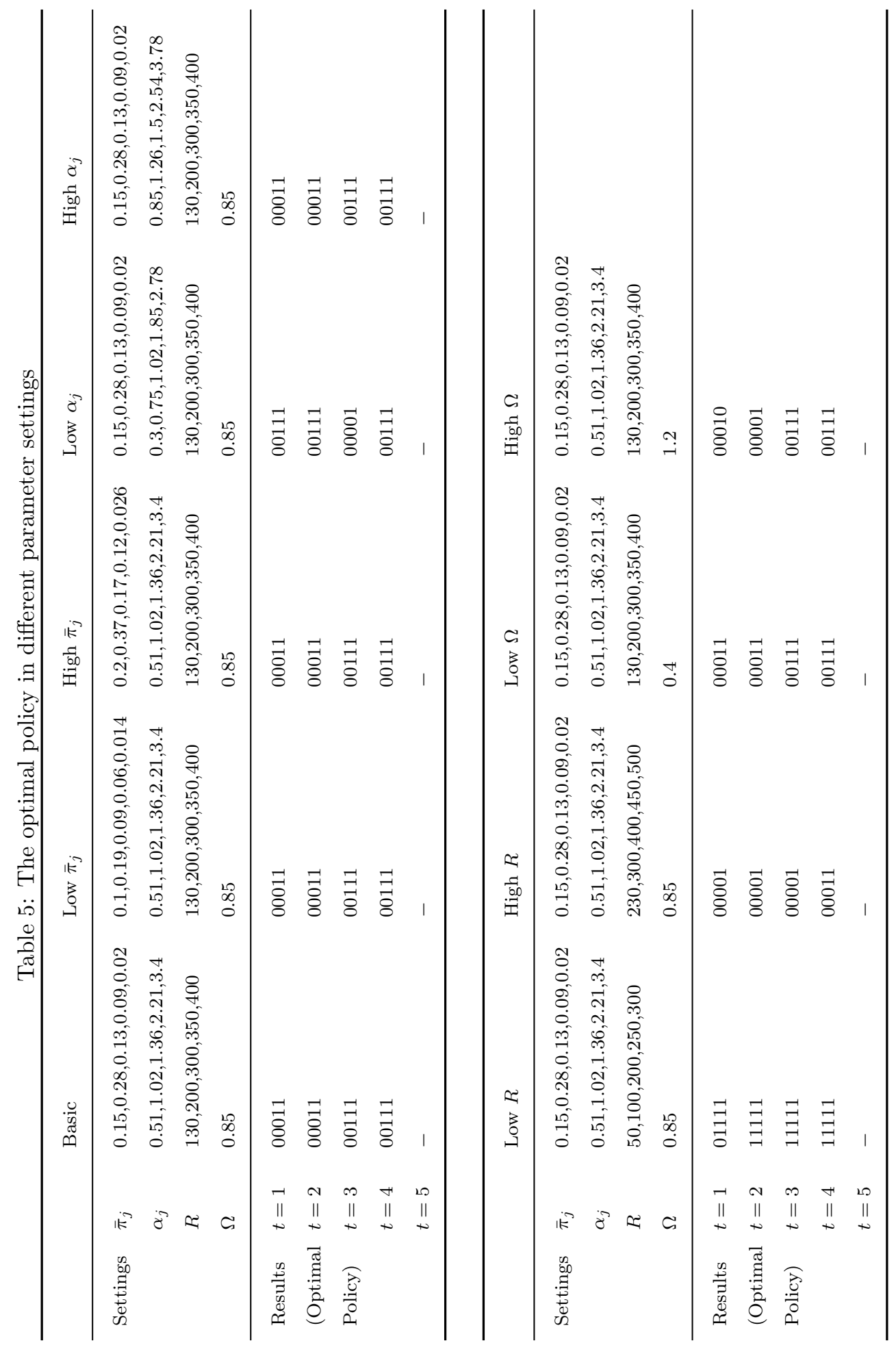


Table 6: Three reservation price groups

\begin{tabular}{llll}
\hline & $\begin{array}{l}\text { Low Reserva- } \\
\text { tion Price }\end{array}$ & $\begin{array}{l}\text { Medium Reser- } \\
\text { vation Price }\end{array}$ & $\begin{array}{l}\text { High Reserva- } \\
\text { tion Price }\end{array}$ \\
\hline $0 \leq D \leq 200$ & 50 & 130 & 230 \\
$200<D \leq 400$ & 100 & 200 & 300 \\
$400<D \leq 800$ & 200 & 300 & 400 \\
$800<D \leq 1500$ & 250 & 350 & 450 \\
$1500<D \leq 2000$ & 300 & 400 & 500 \\
\hline
\end{tabular}
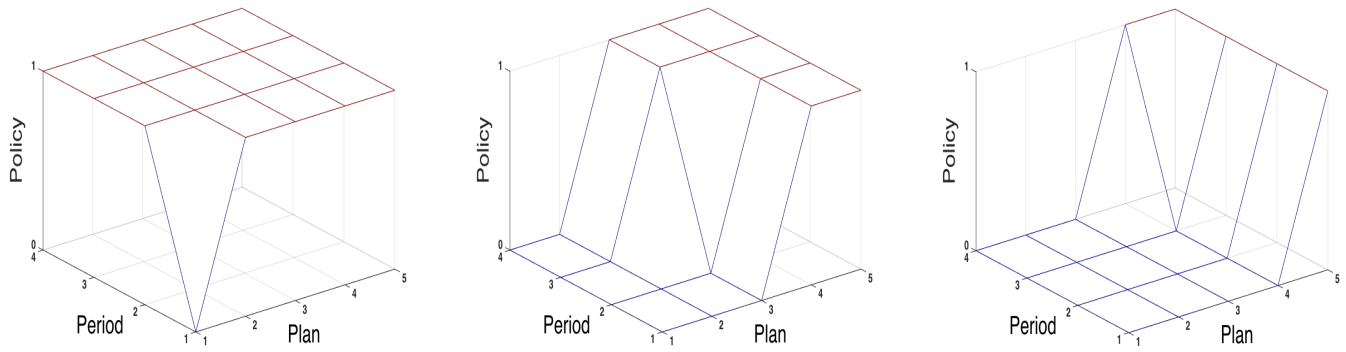
(a) Low reservation price
(b) Medium reservation price
(c) High reservation price

Figure 5: The optimal policy under different reservation price 
Table 7: The effect of total alternative plan number $J$

\begin{tabular}{|c|c|c|c|c|c|c|}
\hline \multirow[b]{2}{*}{$t$} & \multicolumn{3}{|c|}{$J=5$} & \multicolumn{3}{|c|}{$J=8$} \\
\hline & Net Add-in Rate & $I$ & Total Revenue & Net Add-in Rate & $I$ & Total Revenue \\
\hline 1 & 0.32980751 & 00111 & & 0.32980831 & 00111001 & \\
\hline 2 & -0.074382965 & 00111 & 307.402 & -0.165763296 & 00111001 & 358.496 \\
\hline \multirow[t]{2}{*}{3} & - & - & & - & - & \\
\hline & \multicolumn{3}{|c|}{$J=8^{\prime}$} & \multicolumn{3}{|c|}{$J=10$} \\
\hline$T$ & Net Add-in Rate & $I$ & Total Revenue & Net Add-in Rate & $I$ & Total Revenue \\
\hline 1 & 0.25890691 & 00111111 & & 0.120386672 & 0011100111 & \\
\hline 2 & -0.019432071 & 00111111 & 387.967 & 0.04320533 & 0011100111 & 412.569 \\
\hline 3 & - & - & & - & - & \\
\hline
\end{tabular}

along with the growth of $J$. Hence, the SP should add new alternative plans to increase $J$ at times instead of always opening old plans. In addition, the revenue growth of " $J=8^{\prime \prime}$ " group, which added three high-value plans, is faster than " $J=8$ " group, which added three low-value plans. That means if the reservation price allows, adding new high-value plans in total alternative plans is more profitable than adding new low-value plans.

\section{Conclusion}

In wireless communications, the traditional goal of mobile service providers is to attract new customers. However, in present data service-dominated times, service providers should pay more attention to data service because it occupies much more network capacity than voice service. Successful marketing strategies may attract many new customers, but they may also cause the data network to be congested and lower the customer satisfaction level. For this reason, "volume increases but revenue does not increase" and "large inflows and large outflows" phenomena are prevalent in the industry.

Motivated by these challenges, we build a dynamic cell phone plan control model where the service provider can decide a subset of plans to open at the beginning of 
each period. New customers can only subscribe open plans. This dynamic control allows the service provider to dynamically adjust data network utilization and thus achieve high customer satisfaction. In this way, the mobile service provider's revenue is maximized in the long term. The dynamic control problem is a stochastic programming problem where the system state has high dimensions. We analyze the limiting case when the number of customers approaches positive infinity and transform the stochastic dynamic programming to an equivalent integer linear programming formulation.

Moreover, we apply the method to a large mobile service provider in Asia and find that dynamic control can increase its total revenue and improve net add-in rate. Two baffling phenomena can be properly mitigated in this way. In addition, numerical evidence suggests that mobile service providers with low market share benefit more from the dynamic data plan control. Furthermore, the mobile service provider should only open few high-value plans if competitors' price is high. Otherwise, the service provider needs to open more and low-value plans.

Our analysis opens up several opportunities for future research. One natural extension concerns duopoly model, which is common in wireless communications. It would be more interesting to study the optimal control of data plans in a setting with multiple competing service providers. In addition, we allow customers to subscribe and unsubscribe without any costs in each period. A more realistic problem would consider a customer's self-planning problem. Besides, we only get the usage data of customers but don't have the access to the real revenue data. If the real revenue data of the service provider can be gained in future, it is better to make a comparison between the result based on the real revenue data and the result based on our dynamic plan control model to make our conclusions more convincing. 


\section{Acknowledgement}

The authors thank the associate editor and anonymous reviewers for helpful comments and suggestions. This research was supported in part by National Science Foundation [Grant CMMI 1265671], the National Science Foundation of China [Grants 71210002, 71332005 and 71401086] and the Fundamental Research Funds for the Central Universities.

\section{Appendix}

Proof of Proposition 1. We prove this result by induction. At the last period $t=T$,

$$
\begin{aligned}
\lim _{N \rightarrow \infty} \frac{V_{T}\left(\boldsymbol{X}_{T}\right)}{N} & =\lim _{N \rightarrow \infty} \frac{\sum_{j=1}^{J} X_{j, T} \cdot \mathbb{E}\left\{b_{j}+c_{j}\left[\tilde{D}-a_{j}\right]^{+}\right\}}{N} \\
& =\sum_{j=1}^{J} \lim _{N \rightarrow \infty} \frac{X_{j, T}}{N} \cdot \mathbb{E}\left\{b_{j}+c_{j}\left[\tilde{D}-a_{j}\right]^{+}\right\} \\
& =\sum_{j=1}^{J} \pi_{j, T} \cdot \mathbb{E}\left\{b_{j}+c_{j}\left[\tilde{D}-a_{j}\right]^{+}\right\} \\
& =\bar{V}_{T}\left(\boldsymbol{\pi}_{T}\right) .
\end{aligned}
$$

We proceed to the induction stage. Assume

$$
\lim _{N \rightarrow \infty} \frac{V_{t+1}\left(\boldsymbol{X}_{t+1}\right)}{N}=\bar{V}_{t+1}\left(\boldsymbol{\pi}_{t+1}\right),
$$


then

$$
\begin{aligned}
& \lim _{N \rightarrow \infty} \frac{V_{t}\left(\boldsymbol{X}_{t}\right)}{N} \\
= & \lim _{N \rightarrow \infty} \frac{\sum_{j=1}^{J} X_{j, t} \cdot \mathbb{E}\left\{b_{j}+c_{j}\left[\tilde{D}-a_{j}\right]^{+}\right\}}{N} \\
+ & \lim _{N \rightarrow \infty} \frac{\max _{\boldsymbol{I} \in\{0,1\}^{J}}\left\{\sum_{j=1}^{J} \mathbb{E}\left(\tilde{Y}_{j, t}\right) \cdot \mathbb{E}\left\{b_{j}+c_{j}\left[\tilde{D}-a_{j}\right]^{+}\right\}+\mathbb{E}\left[V_{t+1}\left(\tilde{\boldsymbol{X}}_{t+1}\right)\right]\right\}}{N} \\
= & \sum_{j=1}^{J} \pi_{j, t} \cdot \mathbb{E}\left\{b_{j}+c_{j}\left[\tilde{D}-a_{j}\right]^{+}\right\} \\
+ & \max _{\boldsymbol{I}_{t} \in\{0,1\}^{J}}\left\{\lim _{N \rightarrow \infty} \frac{\sum_{j=1}^{J} \mathbb{E}\left(\tilde{Y}_{j, t}\right) \cdot \mathbb{E}\left\{b_{j}+c_{j}\left[\tilde{D}-a_{j}\right]^{+}\right\}+\mathbb{E}\left[V_{t+1}\left(\tilde{\boldsymbol{X}}_{t+1}\right)\right]}{N}\right\} .
\end{aligned}
$$

We have

$$
\begin{aligned}
& \lim _{N \rightarrow \infty} \frac{\sum_{j=1}^{J} \mathbb{E}\left(\widetilde{Y}_{j, t}\right) \cdot \mathbb{E}\left\{b_{j}+c_{j}\left[\tilde{D}-a_{j}\right]^{+}\right\}}{N} \\
= & \lim _{N \rightarrow \infty} \frac{\left(N-\sum_{j=1}^{J} X_{j, t}\right) \cdot \sum_{j=1}^{J} p_{j, t} \cdot \mathbb{E}\left\{b_{j}+c_{j}\left[\tilde{D}-a_{j}\right]^{+}\right\}}{N} \\
= & \left(1-\sum_{j=1}^{J} \pi_{j, t}\right) \cdot \sum_{j=1}^{J} p_{j, t} \cdot \mathbb{E}\left\{b_{j}+c_{j}\left[\tilde{D}-a_{j}\right]^{+}\right\} .
\end{aligned}
$$

Further, the continuity of $\pi_{j, T}$, the continuity of the objective function $V_{t+1} / N$, and the discreteness of the decision space (i.e., $\boldsymbol{I}_{t} \in\{0,1\}^{J}$ ) together guarantee the validness of exchanging the order of max and limit,

$$
\lim _{N \rightarrow \infty} \frac{\mathbb{E}\left[V_{t+1}\left(\tilde{\boldsymbol{X}}_{t+1}\right)\right]}{N}=\mathbb{E}\left[\lim _{N \rightarrow \infty} \frac{V_{t+1}\left(\tilde{\boldsymbol{X}}_{t+1}\right)}{N}\right]=\mathbb{E}\left[\bar{V}_{t+1}\left(\boldsymbol{\pi}_{t+1}\right)\right] .
$$

Due to Strong Law of Large Number,

$$
\begin{aligned}
\pi_{j, t+1} & =\lim _{N \rightarrow \infty} \frac{\tilde{X}_{j, t+1}}{N} \\
& =\lim _{N \rightarrow \infty} \frac{X_{j, t}+\tilde{Y}_{j, t}-\tilde{L}_{j, t}}{N} \\
& =\pi_{j, t} \cdot\left(1-\theta_{j, t}\right)+\left(1-\sum_{j=1}^{J} \pi_{j, t}\right) \cdot p_{j, t}, \forall 1 \leq j \leq J .
\end{aligned}
$$


And

$$
\begin{aligned}
\pi_{0, t+1} & =1-\sum_{j=1}^{J} \pi_{j, t+1} \\
& =\left(1-\sum_{j=1}^{J} p_{j, t}\right) \cdot\left(1-\sum_{j=1}^{J} \pi_{j, t}\right)+\sum_{j=1}^{J} \pi_{j, t} \cdot \theta_{j, t} \\
& =\left(1-\sum_{j=1}^{J} p_{j, t}\right) \cdot \pi_{0, t}+\sum_{j=1}^{J} \pi_{j, t} \cdot \theta_{j, t} .
\end{aligned}
$$

In vector forms, we have $\boldsymbol{\pi}_{t+1}=\boldsymbol{A}_{t}\left(\boldsymbol{I}_{t}, \boldsymbol{\pi}_{t}\right) \cdot \boldsymbol{\pi}_{t}$. By induction, $\lim _{N \rightarrow \infty} \mathbb{E}\left[V_{t+1}\left(\tilde{\boldsymbol{X}}_{t+1}\right)\right] / N=$ $\bar{V}_{t+1}\left(\boldsymbol{A}_{t}\left(\boldsymbol{I}_{t}, \boldsymbol{\pi}_{t}\right) \cdot \boldsymbol{\pi}_{t}\right)$.

To sum up, we have

$$
\begin{aligned}
& \lim _{N \rightarrow \infty} \frac{V_{t}\left(\boldsymbol{X}_{t}\right)}{N} \\
= & \sum_{j=1}^{J} \pi_{j, t} \cdot \mathbb{E}\left\{b_{j}+c_{j}\left[\tilde{D}-a_{j}\right]^{+}\right\} \\
+ & \max _{\boldsymbol{I}_{t} \in\{0,1\}^{J}}\left\{\left(1-\sum_{j=1}^{J} \pi_{j, t}\right) \cdot \sum_{j=1}^{J}\left(p_{j, t} \cdot \mathbb{E}\left\{b_{j}+c_{j}\left[\tilde{D}-a_{j}\right]^{+}\right\}\right)+\bar{V}_{t+1}\left(\boldsymbol{A}_{t}\left(\boldsymbol{I}_{t}, \boldsymbol{\pi}_{t}\right) \cdot \boldsymbol{\pi}_{t}\right)\right\} \\
= & \bar{V}_{t}\left(\boldsymbol{\pi}_{t}\right)
\end{aligned}
$$

for any $1 \leq t<T$.

Proof of Proposition 2. The proof consists of four steps. The first two steps show that $z_{p}(\overline{\boldsymbol{\pi}}) \geq \bar{V}_{1}(\overline{\boldsymbol{\pi}})$, and the last two steps show that $\bar{V}_{1}(\overline{\boldsymbol{\pi}}) \geq z_{p}(\overline{\boldsymbol{\pi}})$.

Step 1. Given that $\left(\boldsymbol{\pi}^{*}, \boldsymbol{I}^{*}\right)$ is the optimal solution to (A), there exist $\boldsymbol{L}^{*}, \boldsymbol{K}_{\boldsymbol{I}}^{*}$ and $\boldsymbol{\Pi}_{\boldsymbol{I}}^{*}$ such that $\left(\boldsymbol{\pi}^{*}, \boldsymbol{L}^{*}, \boldsymbol{K}_{\boldsymbol{I}}^{*}, \boldsymbol{\Pi}_{\boldsymbol{I}}^{*}\right)$ is a feasible solution to (B).

We define $\boldsymbol{\pi}^{*}$ as the unique optimal solution to (A) or one of the optimal solutions to (A), and we denote $\boldsymbol{I}_{t}^{*}, \forall 1 \leq t<T$ as the optimal plan control. Then,

$$
\begin{aligned}
& \boldsymbol{\pi}_{t+1}^{*}=\boldsymbol{A}_{t}\left(\boldsymbol{I}_{t}^{*}, \boldsymbol{\pi}_{t}^{*}\right) \cdot \boldsymbol{\pi}_{t}^{*}, \forall 1 \leq t<T, \\
& 0 \leq \pi_{j, t}^{*} \leq 1, \forall 1 \leq j \leq J, 1 \leq t \leq T, \\
& 0 \leq \sum_{j=1}^{J} \pi_{j, t}^{*} \leq 1, \forall 1 \leq t \leq T, \\
& \pi_{j, 1}^{*}=\bar{\pi}_{j}, \quad \forall 1 \leq j \leq J
\end{aligned}
$$


Recall the definitions of $S\left(\boldsymbol{I}_{t}^{*}, 1\right)$ and $S\left(\boldsymbol{I}_{t}^{*}, 0\right)$, we have

$$
\boldsymbol{\pi}_{t+1}^{*}= \begin{cases}S\left(\boldsymbol{I}_{t}^{*}, 1\right) \cdot \boldsymbol{\pi}_{t}^{*} & \left(\text { if } \sum_{j=1}^{J} \alpha_{j} \cdot \pi_{j, t}^{*} \geq \Omega\right) \\ S\left(\boldsymbol{I}_{t}^{*}, 0\right) \cdot \boldsymbol{\pi}_{t}^{*} & \left(\text { if } \sum_{j=1}^{J} \alpha_{j} \cdot \pi_{j, t}^{*}<\Omega\right)\end{cases}
$$

We construct $\boldsymbol{L}^{*}$ as follows. If $\sum_{j=1}^{J} \alpha_{j} \cdot \pi_{j, t}^{*} \geq \Omega, L_{t}^{*}=1$, otherwise, $L_{t}^{*}=0$. We infer that $\left(\boldsymbol{\pi}^{*}, \boldsymbol{I}_{t}^{*}, \boldsymbol{L}^{*}\right)$ is a feasible solution to

$$
\begin{aligned}
\boldsymbol{\pi}_{t+1} & \geq S\left(\boldsymbol{I}_{t}, 1\right) \cdot \boldsymbol{\pi}_{t}-M \cdot\left(1-L_{t}\right), \quad 1 \leq t<T, \\
\boldsymbol{\pi}_{t+1} & \geq S\left(\boldsymbol{I}_{t}, 0\right) \cdot \boldsymbol{\pi}_{t}-M \cdot L_{t}, \quad 1 \leq t<T, \\
-M \cdot\left(1-L_{t}\right) & \leq \sum_{j=1}^{J} \alpha_{j} \cdot \pi_{j, t}-\Omega \leq M \cdot L_{t}, \quad 1 \leq t<t .
\end{aligned}
$$

We construct $\boldsymbol{K}_{\boldsymbol{I}}^{*}$ as follows. For all $\boldsymbol{I}_{t} \in\{0,1\}^{J}$, if $\boldsymbol{I}_{t}=\boldsymbol{I}_{t}^{*}, K_{\boldsymbol{I}_{t}, t}^{*}=1$, otherwise, $K_{\boldsymbol{I}_{t}, t}^{*}=0$. We also construct $\Pi_{\boldsymbol{I}}^{*}$ as $\Pi_{\boldsymbol{I}_{t}, t}^{*}=\pi_{0, t}^{*} \cdot K_{\boldsymbol{I}_{t}, t}^{*}, \forall \boldsymbol{I}_{t} \in\{0,1\}^{J}$. We further get rid of $\boldsymbol{I}_{t}^{*}$ in constraints (3) and argue that $\left(\boldsymbol{\pi}^{*}, \boldsymbol{L}^{*}, \boldsymbol{K}_{\boldsymbol{I}}^{*}, \boldsymbol{\Pi}_{\boldsymbol{I}}^{*}\right)$ is a feasible solution to

$$
\begin{aligned}
& \boldsymbol{\pi}_{t+1} \geq S\left(\boldsymbol{I}_{t}, 1\right) \cdot \boldsymbol{\pi}_{t}-M \cdot\left(1-L_{t}\right)-M \cdot\left(1-K_{\boldsymbol{I}_{t}, t}\right), \forall \boldsymbol{I}_{t} \in\{0,1\}^{J}, 1 \leq t<T, \\
& \boldsymbol{\pi}_{t+1} \geq S\left(\boldsymbol{I}_{t}, 0\right) \cdot \boldsymbol{\pi}_{t}-M \cdot L_{t}-M \cdot\left(1-K_{\boldsymbol{I}_{t}, t}\right), \forall \boldsymbol{I}_{t} \in\{0,1\}^{J}, 1 \leq t<T, \\
& \sum_{\boldsymbol{I}_{t} \in\{0,1\}^{J}} K_{\boldsymbol{I}_{t}, t}=1, \forall 1 \leq t<T, \quad K_{\boldsymbol{I}_{t}, t} \in\{0,1\}, \forall \boldsymbol{I}_{t} \in\{0,1\}^{J}, \forall 1 \leq t<T, \\
& \Pi_{\boldsymbol{I}_{t}, t} \leq \pi_{0, t}, \forall 1 \leq t<T, \boldsymbol{I}_{t} \in\{0,1\}^{J}, \quad \Pi_{\boldsymbol{I}_{t}, t} \leq K_{\boldsymbol{I}_{t}, t}, \forall 1 \leq t<T, \boldsymbol{I}_{t} \in\{0,1\}^{J} .
\end{aligned}
$$

We conclude that $\left(\boldsymbol{\pi}^{*}, \boldsymbol{L}^{*}, \boldsymbol{K}_{\boldsymbol{I}}^{*}, \boldsymbol{\Pi}_{\boldsymbol{I}}^{*}\right)$ satisfies the constraints in (B), so $\left(\boldsymbol{\pi}^{*}, \boldsymbol{L}^{*}, \boldsymbol{K}_{\boldsymbol{I}}^{*}, \boldsymbol{\Pi}_{\boldsymbol{I}}^{*}\right)$ is a feasible solution of $(\mathrm{B})$.

Step 2. $z_{p}(\overline{\boldsymbol{\pi}}) \geq \bar{V}_{1}(\overline{\boldsymbol{\pi}})$.

Because $\left(\boldsymbol{\pi}^{*}, \boldsymbol{I}^{*}\right)$ is the optimal solution to (A) and $\left(\boldsymbol{\pi}^{*}, \boldsymbol{L}^{*}, \boldsymbol{K}_{\boldsymbol{I}}^{*}, \boldsymbol{\Pi}_{\boldsymbol{I}}^{*}\right)$ is a feasible 
solution to (B),

$$
\begin{aligned}
\bar{V}_{1}(\overline{\boldsymbol{\pi}}) & =\sum_{j=1}^{J} \pi_{j, 1}^{*} \cdot \mathbb{E}\left\{b_{j}+c_{j} \cdot\left[\tilde{D}-a_{j}\right]^{+}\right\} \\
& +\left(1-\sum_{j=1}^{J} \pi_{j, 1}^{*}\right) \sum_{j=1}^{J} p_{j, 1}\left(\boldsymbol{I}_{1}^{*}\right) \cdot \mathbb{E}\left\{b_{j}+c_{j} \cdot\left[\tilde{D}-a_{j}\right]^{+}\right\}+\bar{V}_{2}\left(\boldsymbol{\pi}_{2}^{*}\right) \\
& =\sum_{t=1}^{T} \sum_{j=1}^{J} \pi_{j, t}^{*} \cdot \mathbb{E}\left\{b_{j}+c_{j} \cdot\left[\tilde{D}-a_{j}\right]^{+}\right\} \\
& +\sum_{t=1}^{T-1}\left(1-\sum_{j=1}^{J} \pi_{j, t}^{*}\right) \sum_{j=1}^{J} p_{j, t}\left(\boldsymbol{I}_{t}^{*}\right) \cdot \mathbb{E}\left\{b_{j}+c_{j} \cdot\left[\tilde{D}-a_{j}\right]^{+}\right\} \quad \text { (by recursion) } \\
& =\sum_{t=1}^{T} \sum_{j=1}^{J} \pi_{j, t}^{*} \cdot \mathbb{E}\left\{b_{j}+c_{j} \cdot\left[\tilde{D}-a_{j}\right]^{+}\right\} \\
& +\sum_{t=1}^{T-1} \sum_{j=1}^{J} \pi_{0, t}^{*} \sum_{\boldsymbol{I}_{t} \in\{0,1\}^{J}} p_{j, t}\left(\boldsymbol{I}_{t}\right) \cdot K_{\boldsymbol{I}_{t}, t}^{*} \cdot \mathbb{E}\left\{b_{j}+c_{j} \cdot\left[\tilde{D}-a_{j}\right]^{+}\right\} \\
& \left.=\sum_{t=1}^{T} \sum_{j=1}^{J} \pi_{j, t}^{*} \cdot \mathbb{E}\left\{b_{j}+c_{j} \cdot\left[\tilde{D}-a_{j}\right]^{+}\right\} \quad \text { by definition of } \pi_{0, t}^{*}, K_{\boldsymbol{I}_{t}, t}^{*}\right) \\
& +\sum_{t=1}^{T-1} \sum_{j=1}^{J} \sum_{\boldsymbol{I}_{t} \in\{0,1\}^{J}} p_{j, t}\left(\boldsymbol{I}_{t}\right) \cdot \Pi_{\boldsymbol{I}_{t}, t}^{*} \cdot \mathbb{E}\left\{b_{j}+c_{j} \cdot\left[\tilde{D}-a_{j}\right]^{+}\right\} .
\end{aligned}
$$

(by definition of $\Pi_{\boldsymbol{I}_{t, t}}^{*}$ )

Since $\left(\boldsymbol{\pi}^{*}, \boldsymbol{L}^{*}, \boldsymbol{K}_{\boldsymbol{I}}^{*}, \boldsymbol{\Pi}_{\boldsymbol{I}}^{*}\right)$ is a feasible solution to (B),

$$
\begin{aligned}
z_{p}(\overline{\boldsymbol{\pi}}) & \geq \sum_{t=1}^{T} \sum_{j=1}^{J} \pi_{j, t}^{*} \cdot \mathbb{E}\left\{b_{j}+c_{j} \cdot\left[\tilde{D}-a_{j}\right]^{+}\right\} \\
& +\sum_{t=1}^{T-1} \sum_{j=1}^{J} \sum_{\boldsymbol{I}_{t} \in\{0,1\}^{J}} p_{j, t}\left(\boldsymbol{I}_{t}\right) \cdot \Pi_{\boldsymbol{I}_{t}, t}^{*} \cdot \mathbb{E}\left\{b_{j}+c_{j} \cdot\left[\tilde{D}-a_{j}\right]^{+}\right\} \\
& =\bar{V}_{1}(\overline{\boldsymbol{\pi}}) .
\end{aligned}
$$

Step 3. Given that $\left(\hat{\boldsymbol{\pi}}, \hat{\boldsymbol{L}}, \hat{\boldsymbol{K}}_{\boldsymbol{I}}, \hat{\boldsymbol{\Pi}}_{\boldsymbol{I}}\right)$ is the optimal solution to (B), there exists $\hat{\boldsymbol{I}}$ such that $(\hat{\boldsymbol{\pi}}, \hat{\boldsymbol{I}})$ is a feasible solution to $(\mathrm{A})$.

If we define $\left(\hat{\boldsymbol{\pi}}, \hat{\boldsymbol{L}}, \hat{\boldsymbol{K}}_{\boldsymbol{I}}, \hat{\boldsymbol{\Pi}}_{\boldsymbol{I}}\right)$ as the unique optimal solution or one of the optimal 
solutions to (B), then $\left(\hat{\boldsymbol{\pi}}, \hat{\boldsymbol{L}}, \hat{\boldsymbol{K}}_{\boldsymbol{I}}, \hat{\boldsymbol{\Pi}}_{\boldsymbol{I}}\right)$ is a feasible solution to (B):

$$
\begin{aligned}
& \hat{\boldsymbol{\pi}}_{t+1} \geq S\left(\boldsymbol{I}_{t}, 1\right) \cdot \hat{\boldsymbol{\pi}}_{t}-M \cdot\left(1-\hat{K}_{\boldsymbol{I}_{t}, t}\right)-M \cdot\left(1-\hat{L}_{t}\right), \forall \boldsymbol{I}_{t} \in\{0,1\}^{J}, 1 \leq t<T, \\
& \hat{\boldsymbol{\pi}}_{t+1} \geq S\left(\boldsymbol{I}_{t}, 0\right) \cdot \hat{\boldsymbol{\pi}}_{t}-M \cdot\left(1-\hat{K}_{\boldsymbol{I}_{t}, t}\right)-M \cdot \hat{L}_{t}, \forall \boldsymbol{I}_{t} \in\{0,1\}^{J}, 1 \leq t<T, \\
& \sum_{j=0}^{J} \hat{\pi}_{j, t}=1, \forall 1 \leq t \leq T, \quad 0 \leq \hat{\pi}_{j, t} \leq 1, \forall 1 \leq j \leq J, 1 \leq t \leq T, \\
& \sum_{\boldsymbol{I}_{t} \in\{0,1\}^{J}} \hat{K}_{\boldsymbol{I}_{t}, t}=1, \forall 1 \leq t<T, \quad \hat{K}_{\boldsymbol{I}_{t}, t} \in\{0,1\}, \forall \boldsymbol{I}_{t} \in\{0,1\}^{J}, 1 \leq t<T, \\
& -M \cdot\left(1-\hat{L}_{t}\right) \leq \sum_{j=1}^{J} \alpha_{j} \cdot \hat{\pi}_{j, t}-\Omega \leq M \cdot \hat{L}_{t}, \forall 1 \leq t<T \\
& \hat{L}_{t} \in\{0,1\}, \forall 1 \leq t<T .
\end{aligned}
$$

Define $\hat{\boldsymbol{I}}_{t}$ as the $\boldsymbol{I}_{t}$ such that $\hat{K}_{\hat{\boldsymbol{I}}_{t}, t}=1$. If $\sum_{j=1}^{J} \alpha_{j} \cdot \hat{\pi}_{j, t} \geq \Omega$, then (9) and (10) infer that $\hat{L}_{t}=1$. When $\boldsymbol{I}_{t}=\hat{\boldsymbol{I}}_{t},(5)$ becomes $\hat{\boldsymbol{\pi}}_{t+1} \geq S\left(\hat{\boldsymbol{I}}_{t}, 1\right) \cdot \hat{\boldsymbol{\pi}}_{t}$. Because of $(7)$, we have $\hat{\boldsymbol{\pi}}_{t+1}=S\left(\hat{\boldsymbol{I}}_{t}, 1\right) \cdot \hat{\boldsymbol{\pi}}_{t}$. Similarly, if $\sum_{j=1}^{J} \alpha_{j} \cdot \hat{\pi}_{j, t}<\Omega$, we must have $\hat{L}_{t}=0$ and $\hat{\boldsymbol{\pi}}_{t+1}=S\left(\hat{\boldsymbol{I}}_{t}, 0\right) \cdot \hat{\boldsymbol{\pi}}_{t}$. By definition of $\boldsymbol{A}\left(\boldsymbol{I}_{t}, \boldsymbol{\pi}_{t}\right),\left(\hat{\boldsymbol{\pi}}_{t}, \hat{\boldsymbol{I}}_{t}\right)$ satisfies

$$
\hat{\boldsymbol{\pi}}_{t+1}=\boldsymbol{A}\left(\hat{\boldsymbol{I}}_{t}, \hat{\boldsymbol{\pi}}_{t}\right) \cdot \hat{\boldsymbol{\pi}}_{t}, \forall 1 \leq t<T
$$

In particular, constraint (11) is

$$
\begin{aligned}
\hat{\pi}_{j, t+1} & =p_{j, t}\left(\hat{\boldsymbol{I}}_{t}\right) \cdot \hat{\pi}_{0, t}+\left(1-\theta_{j, t}\left(\hat{\boldsymbol{\pi}}_{t}\right)\right) \cdot \hat{\pi}_{j, t} \\
& =p_{j, t}\left(\hat{\boldsymbol{I}}_{t}\right)\left(1-\sum_{i=1}^{J} \hat{\pi}_{i, t}\right)+\left(1-\theta_{j, t}\left(\hat{\boldsymbol{\pi}}_{t}\right)\right) \cdot \hat{\pi}_{j, t} \\
& =p_{j, t}\left(\hat{\boldsymbol{I}}_{t}\right)+\left(1-\theta_{j, t}\left(\hat{\boldsymbol{\pi}}_{t}\right)-p_{j, t}\left(\hat{\boldsymbol{I}}_{t}\right)\right) \cdot \hat{\pi}_{j, t}-p_{j, t}\left(\hat{\boldsymbol{I}}_{t}\right) \cdot \sum_{i \neq j} \hat{\pi}_{i, t} \\
& \left.=\sum_{i=0}^{J} \boldsymbol{A}_{t}\left(\hat{\boldsymbol{I}}, \hat{\boldsymbol{\pi}}_{t}\right) \cdot \hat{\pi}_{i, t} \quad \text { (by definitions of } \boldsymbol{A}\left(\boldsymbol{I}_{t}, \boldsymbol{\pi}\right)\right)
\end{aligned}
$$

To conclude, $\left(\hat{\boldsymbol{\pi}}_{t}, \hat{\boldsymbol{I}}_{t}\right)$ satisfies

$$
\hat{\boldsymbol{\pi}}_{t+1}=\boldsymbol{A}_{t}\left(\hat{\boldsymbol{I}}_{t}, \hat{\boldsymbol{\pi}}_{t}\right) \cdot \hat{\boldsymbol{\pi}}_{t}
$$

Because $\sum_{j=0}^{J} \hat{\pi}_{j, t}=1, \forall 1 \leq t \leq T$ and $0 \leq \hat{\pi}_{j, t} \leq 1, \forall 1 \leq j \leq J, 1 \leq t \leq T$, we have

$$
0 \leq \sum_{j=1}^{J} \hat{\pi}_{j, t} \leq 1, \forall 1 \leq t \leq T .
$$


Combining constraints (12) and (13), we find that $(\hat{\boldsymbol{\pi}}, \hat{\boldsymbol{I}})$ satisfies the constraints in (A). So $(\hat{\boldsymbol{\pi}}, \hat{\boldsymbol{I}})$ is a feasible solution to $(\mathrm{A})$.

Step 4. $\bar{V}_{1}(\overline{\boldsymbol{\pi}}) \geq z_{p}(\overline{\boldsymbol{\pi}})$.

We prove this result by induction. When $T=1$

$$
\begin{array}{rlrl}
z_{p}(\overline{\boldsymbol{\pi}}) & =\sum_{j=1}^{J} \hat{\pi}_{j, 1} \cdot \mathbb{E}\left\{b_{j}+c_{j} \cdot\left[\tilde{D}-a_{j}\right]^{+}\right\} \\
& =\bar{V}_{1}(\overline{\boldsymbol{\pi}}) . & \text { (by definition of } \left.\bar{V}_{1}(\overline{\boldsymbol{\pi}})\right)
\end{array}
$$

When $T \leq \tau$, assume that $\bar{V}_{1}(\overline{\boldsymbol{\pi}}) \geq z_{p}(\overline{\boldsymbol{\pi}})$. We proceed to the case where $T=\tau+1$. By induction, we have

$$
\begin{aligned}
\bar{V}_{2}\left(\hat{\boldsymbol{\pi}}_{2}\right) & \geq \sum_{t=2}^{T} \sum_{j=1}^{J} \hat{\pi}_{j, t} \cdot \mathbb{E}\left\{b_{j}+c_{j} \cdot\left[\tilde{D}-a_{j}\right]^{+}\right\} \\
& +\sum_{t=2}^{T-1} \sum_{j=1}^{J}\left(1-\sum_{i=1}^{J} \hat{\pi}_{i, t}\right) \cdot p_{j, t}\left(\hat{\boldsymbol{I}}_{t}\right) \cdot \mathbb{E}\left\{b_{j}+c_{j} \cdot\left[\tilde{D}-a_{j}\right]^{+}\right\} .
\end{aligned}
$$

We prove that

$$
\begin{aligned}
\bar{V}_{1}(\overline{\boldsymbol{\pi}}) & =\max _{\boldsymbol{I}_{1} \in\{0,1\}^{J}}\left\{\sum_{j=1}^{J} \bar{\pi}_{j} \cdot \mathbb{E}\left\{b_{j}+c_{j} \cdot\left[\tilde{D}-a_{j}\right]^{+}\right\}\right. \\
& \left.+\left(1-\sum_{i=1}^{J} \bar{\pi}_{i}\right) \sum_{j=1}^{J} p_{j, 1}\left(\boldsymbol{I}_{1}\right) \cdot \mathbb{E}\left\{b_{j}+c_{j} \cdot\left[\tilde{D}-a_{j}\right]^{+}\right\}+\bar{V}_{2}\left(\boldsymbol{A}_{1}\left(\boldsymbol{I}_{1}, \overline{\boldsymbol{\pi}}\right) \cdot \overline{\boldsymbol{\pi}}\right)\right\} \\
\geq & \sum_{j=1}^{J} \hat{\pi}_{j, 1} \cdot \mathbb{E}\left\{b_{j}+c_{j} \cdot\left[\tilde{D}-a_{j}\right]^{+}\right\}+\hat{\pi}_{0,1} \sum_{j=1}^{J} p_{j, 1}\left(\hat{\boldsymbol{I}}_{1}\right) \cdot \mathbb{E}\left\{b_{j}+c_{j} \cdot\left[\tilde{D}-a_{j}\right]^{+}\right\}+\bar{V}_{2}\left(\hat{\boldsymbol{\pi}}_{2}\right) \\
\geq & \sum_{t=1}^{T} \sum_{j=1}^{J} \hat{\pi}_{j, t} \cdot \mathbb{E}\left\{b_{j}+c_{j} \cdot\left[\tilde{D}-a_{j}\right]^{+}\right\}+\sum_{t=1}^{T-1} \sum_{j=1}^{J} \hat{\pi}_{0, t} \cdot p_{j, t}\left(\hat{\boldsymbol{I}}_{t}\right) \cdot \mathbb{E}\left\{b_{j}+c_{j} \cdot\left[\tilde{D}-a_{j}\right]^{+}\right\} \\
= & \sum_{t=1}^{T} \sum_{j=1}^{J} \hat{\pi}_{j, t} \cdot \mathbb{E}\left\{b_{j}+c_{j} \cdot\left[\tilde{D}-a_{j}\right]^{+}\right\} \\
& +\sum_{t=1}^{T-1} \sum_{j=1}^{J} \sum_{\boldsymbol{I}_{t} \in\{0,1\}^{J}} \hat{\pi}_{0, t} \cdot p_{j, t}\left(\boldsymbol{I}_{t}\right) \cdot \hat{K}_{\boldsymbol{I}_{t}, t} \cdot \mathbb{E}\left\{b_{j}+c_{j} \cdot\left[\tilde{D}-a_{j}\right]^{+}\right\}
\end{aligned}
$$

(by definition of $\hat{K}_{\boldsymbol{I}_{t}, t}$ ) 


$$
\begin{aligned}
& =\sum_{t=1}^{T} \sum_{j=1}^{J} \hat{\pi}_{j, t} \cdot \mathbb{E}\left\{b_{j}+c_{j} \cdot\left[\tilde{D}-a_{j}\right]^{+}\right\} \\
& \left.+\sum_{t=1}^{T-1} \sum_{j=1}^{J} \sum_{\boldsymbol{I}_{t} \in\{0,1\}^{J}} p_{j, t}\left(\boldsymbol{I}_{t}\right) \cdot \hat{\Pi}_{\boldsymbol{I}_{t}, t} \cdot \mathbb{E}\left\{b_{j}+c_{j} \cdot\left[\tilde{D}-a_{j}\right]^{+}\right\} \quad \text { (by definition of } \hat{\Pi}_{\boldsymbol{I}_{t}, t}\right) \\
& =z_{p}(\bar{\pi}) .
\end{aligned}
$$

Thus, we prove that $\bar{V}_{1}(\overline{\boldsymbol{\pi}}) \geq z_{p}(\overline{\boldsymbol{\pi}})$.

In conclusion, we have $\bar{V}_{1}(\overline{\boldsymbol{\pi}})=z_{p}(\overline{\boldsymbol{\pi}})$. Proposition 2 holds.

Proof of Proposition 3. The proof consists of four steps. The first two steps show that $\hat{z}_{p}(\overline{\boldsymbol{\pi}}) \geq z_{p}(\overline{\boldsymbol{\pi}})$, and the last two steps show that $z_{p}(\overline{\boldsymbol{\pi}}) \geq \hat{z}_{p}(\overline{\boldsymbol{\pi}})$.

Step 1. Given that $\left(\boldsymbol{\pi}^{*}, \boldsymbol{L}^{*}, \boldsymbol{K}_{\boldsymbol{I}}^{*}, \boldsymbol{\Pi}_{\boldsymbol{I}}^{*}\right)$ is the optimal solution to (B), there exists $\boldsymbol{\beta}^{*}, \boldsymbol{\gamma}^{*}, \boldsymbol{I}^{*}$ and $\boldsymbol{p} \boldsymbol{p}^{*}$ such that $\left(\boldsymbol{\pi}^{*}, \boldsymbol{L}^{*}, \boldsymbol{\beta}^{*}, \boldsymbol{\gamma}^{*}, \boldsymbol{I}^{*}, \boldsymbol{p} \boldsymbol{p}^{*}\right)$ is a feasible solution to (C).

We define $\left(\boldsymbol{\pi}^{*}, \boldsymbol{L}^{*}, \boldsymbol{K}_{\boldsymbol{I}}^{*}, \boldsymbol{\Pi}_{\boldsymbol{I}}^{*}\right)$ as the unique or one of the optimal solutions to (B), $\left(\boldsymbol{\pi}^{*}, \boldsymbol{L}^{*}, \boldsymbol{K}_{\boldsymbol{I}}^{*}, \boldsymbol{\Pi}_{\boldsymbol{I}}^{*}\right)$ is also a feasible solution to (B) and satisfies the following constraints:

$$
\begin{aligned}
& \boldsymbol{\pi}_{t+1}^{*} \geq S\left(\boldsymbol{I}_{t}, 1\right) \cdot \boldsymbol{\pi}_{t}^{*}-M \cdot\left(1-L_{t}^{*}\right)-M \cdot\left(1-K_{\boldsymbol{I}_{t}, t}^{*}\right), \forall \boldsymbol{I}_{t} \in\{0,1\}^{J}, \\
& \boldsymbol{\pi}_{t+1}^{*} \geq S\left(\boldsymbol{I}_{t}, 0\right) \cdot \boldsymbol{\pi}_{t}^{*}-M \cdot L_{t}^{*}-M \cdot\left(1-K_{\boldsymbol{I}_{t}, t}^{*}\right), \forall \boldsymbol{I}_{t} \in\{0,1\}^{J}, \\
& \sum_{j=0}^{J} \pi_{j, t}^{*}=1, \quad 0 \leq \pi_{j, t}^{*} \leq 1, \forall 1 \leq j \leq J, \\
& \sum_{\boldsymbol{I}_{t} \in\{0,1\}^{J}} K_{\boldsymbol{I}_{t}, t}^{*}=1, \quad K_{\boldsymbol{I}_{t}, t}^{*} \in\{0,1\}, \forall \boldsymbol{I}_{t} \in\{0,1\}^{J}, \\
& -M \cdot\left(1-L_{t}^{*}\right) \leq \sum_{j=1}^{J} \alpha_{j} \cdot \pi_{j, t}^{*}-\Omega \leq M \cdot L_{t}^{*}, \\
& L_{t}^{*} \in\{0,1\} .
\end{aligned}
$$

We construct $\boldsymbol{I}_{t}^{*}$ as $\boldsymbol{I}_{t}^{*}=\sum_{\boldsymbol{I}_{t} \in\{0,1\}^{J}} \boldsymbol{I}_{t} \cdot K_{\boldsymbol{I}_{t}, t}^{*}$. Obviously, when $\boldsymbol{I}_{t}=\boldsymbol{I}_{t}^{*}, K_{\boldsymbol{I}_{t}, t}^{*}=1$, otherwise, $K_{\boldsymbol{I}_{t}, t}^{*}=0$. Equation (14) suggests that if $K_{\boldsymbol{I}_{t}, t}^{*}=1$ and $L_{t}^{*}=1$, then 
$\boldsymbol{\pi}_{t+1}^{*} \geq S\left(\boldsymbol{I}_{t}^{*}, 1\right) \cdot \boldsymbol{\pi}_{t}^{*}$. In particular, $\boldsymbol{\pi}^{*}$ satisfies

$$
\begin{aligned}
\pi_{j, t+1}^{*} & \geq S\left(\boldsymbol{I}_{t}^{*}, 1\right)_{j, 0} \cdot \pi_{0, t}^{*}+S\left(\boldsymbol{I}_{t}^{*}, 1\right)_{j, j} \cdot \pi_{j, t}^{*} \\
& =p_{j, t}\left(\boldsymbol{I}_{t}^{*}\right) \cdot \pi_{0, t}^{*}+\left(1-\bar{\theta}_{j}\right) \cdot \pi_{j, t}^{*}, \forall 1 \leq j \leq J, \\
\pi_{0, t+1}^{*} & \geq S\left(\boldsymbol{I}_{t}^{*}, 1\right)_{j, 0} \cdot \pi_{0, t}^{*}+\sum_{j=1}^{J} S\left(\boldsymbol{I}_{t}^{*}, 1\right)_{j, j} \cdot \pi_{j, t}^{*} \\
& =\left(1-\sum_{j=1}^{J} p_{j, t}\left(\boldsymbol{I}_{t}^{*}\right)\right) \cdot \pi_{0, t}^{*}+\sum_{j=1}^{J} \bar{\theta}_{j} \cdot \pi_{j, t}^{*} .
\end{aligned}
$$

Summing up the two inequations in (20)

$$
\begin{aligned}
\sum_{j=0}^{J} \pi_{j, t+1}^{*} & \geq\left(1-\sum_{j=1}^{J} p_{j, t}\left(\boldsymbol{I}_{t}^{*}\right)\right) \cdot \pi_{0, t}^{*}+\sum_{j=1}^{J} p_{j, t}\left(\boldsymbol{I}_{t}^{*}\right) \cdot \pi_{0, t}^{*}+\sum_{j=1}^{J}\left(1-\bar{\theta}_{j}\right) \cdot \pi_{j, t}^{*}+\sum_{j=1}^{J} \bar{\theta}_{j} \cdot \pi_{j, t}^{*} \\
& =\pi_{0, t}^{*}+\sum_{j=1}^{J} \pi_{j, t}^{*}=\sum_{j=0}^{J} \pi_{j, t}^{*} .
\end{aligned}
$$

Because of (16), we have

$$
\begin{aligned}
& \pi_{j, t+1}^{*}=p_{j, t}\left(\boldsymbol{I}_{t}^{*}\right) \cdot \pi_{0, t}^{*}+\left(1-\bar{\theta}_{j}\right) \cdot \pi_{j, t}^{*}, \forall 1 \leq j \leq J, \\
& \pi_{0, t+1}^{*}=\left(1-\sum_{j=1}^{J} p_{j, t}\left(\boldsymbol{I}_{t}^{*}\right)\right) \cdot \pi_{0, t}^{*}+\sum_{j=1}^{J} \bar{\theta}_{j} \cdot \pi_{j, t}^{*} .
\end{aligned}
$$

Similarly, when $K_{\boldsymbol{I}_{t}, t}^{*}=1$ and $L_{t}^{*}=0,(21)$ becomes

$$
\begin{aligned}
& \pi_{j, t+1}^{*}=\pi_{j, t}^{*}+p_{j, t} \cdot \pi_{0, t}^{*}, \forall 1 \leq j \leq J, \\
& \pi_{0, t+1}^{*}=\pi_{0, t}^{*}-\sum_{j=1}^{J} p_{j, t}\left(\boldsymbol{I}_{t}^{*}\right) \cdot \pi_{0, t}^{*} .
\end{aligned}
$$

We construct $\beta_{j, t}^{*}=p_{j, t}\left(\boldsymbol{I}_{t}^{*}\right) \cdot \pi_{0, t}^{*}$ and

$$
\gamma_{j, t}^{*}= \begin{cases}\bar{\theta}_{j} \cdot \pi_{j, t}^{*}, & \text { if } \sum_{j=1}^{J} \alpha_{j} \cdot \pi_{j, t}^{*} \geq \Omega \\ 0, & \text { if } \sum_{j=1}^{J} \alpha_{j} \cdot \pi_{j, t}^{*}<\Omega .\end{cases}
$$

Given the definitions of $\beta_{j, t}^{*}$ and $\gamma_{j, t}^{*}$, (21) and (22) become

$$
\begin{aligned}
& \pi_{j, t+1}^{*}=\pi_{j, t}^{*}+\beta_{j, t}^{*}-\gamma_{j, t}^{*}, \forall 1 \leq j \leq J, \\
& \pi_{0, t+1}^{*}=\pi_{0, t}^{*}-\sum_{j=1}^{J} \beta_{j, t}^{*}+\sum_{j=1}^{J} \gamma_{j, t}^{*} .
\end{aligned}
$$


We denote $I_{j, t}^{*}$ as the $j$ th element of $\boldsymbol{I}_{t}^{*}$, for $1 \leq j \leq J$. When $I_{j, t}^{*}=0$, by definition of $\beta_{j, t}^{*}$, we have $\beta_{j, t}^{*}=0$. And when $I_{j, t}^{*}=1, \beta_{j, t}^{*}=p_{j, t}\left(\boldsymbol{I}_{t}^{*}\right) \cdot \pi_{0, t}^{*}$. Then $\beta_{j, t}^{*}$ must satisfy:

$$
-M \cdot I_{j, t}^{*} \leq \beta_{j, t} \leq M \cdot I_{j, t}^{*}
$$

By definition of $\gamma_{j, t}^{*}$, when $L_{t}^{*}=1, \gamma_{j, t}^{*}=\bar{\theta}_{j} \cdot \pi_{j, t}^{*}$ and when $L_{t}^{*}=0, \gamma_{j, t}^{*}=0$. Thus, $\gamma_{j, t}^{*}$ satisfies the following constraints:

$$
\begin{gathered}
\pi_{j, t}^{*} \cdot \bar{\theta}_{j}-M\left(1-L_{t}^{*}\right) \leq \gamma_{j, t} \leq \pi_{j, t}^{*} \cdot \bar{\theta}_{j}+M\left(1-L_{t}^{*}\right) \\
-M \cdot L_{t}^{*} \leq \gamma_{j, t} \leq M \cdot L_{t}^{*}
\end{gathered}
$$

Recall the definition of $X_{i, j}, \forall 1 \leq i<j \leq J$, we have

$$
X_{i, j}=\frac{b_{j}-b_{i}}{c_{i}}+a_{i}
$$

Under Assumption 1, there only exists one $X_{i, j}$ for plan $i$ and plan $j$. When the customers' data usage is below $X_{i, j}$, plan $i$ is preferred to plan $j$ by customers. When the data usage is over $X_{i, j}$, the situation reverses. Next, we show that $p_{j, t}\left(\boldsymbol{I}_{t}^{*}\right)$ satisfies constraints (27) to (31).

$$
\begin{aligned}
& p_{j, t}\left(\boldsymbol{I}_{t}^{*}\right) \leq F\left(X_{j, i_{2}}\right)-F\left(X_{i_{1}, j}\right)+M \cdot\left(2-I_{i_{1}, t}^{*}-I_{i_{2}, t}^{*}\right), \forall 1 \leq i_{1}<j<i_{2} \leq J, \\
& p_{j, t}\left(\boldsymbol{I}_{t}^{*}\right) \leq F\left(X_{j, i_{2}}\right)-F\left(X_{0, j}\right)+M \cdot\left(1-I_{i_{2}, t}^{*}\right), \forall 1 \leq j<i_{2} \leq J \\
& p_{j, t}\left(\boldsymbol{I}_{t}^{*}\right) \leq 1-F\left(X_{i_{1}, j}\right)+M \cdot\left(1-I_{i_{1}, t}^{*}\right), \forall 1 \leq i_{1}<j \leq J \\
& p_{j, t}\left(\boldsymbol{I}_{t}^{*}\right) \leq 1-F\left(X_{0, j}\right), \forall 1 \leq j \leq J \\
& p_{0, t}\left(\boldsymbol{I}_{t}^{*}\right) \leq F\left(X_{0, j}\right)+M \cdot\left(1-I_{j, t}^{*}\right), \forall 1 \leq j \leq J
\end{aligned}
$$

Firstly we discuss the constraint (27). If either $I_{i_{1}, t}^{*}=0$ or $I_{i_{2}, t}^{*}=0,(27)$ holds trivially. We can concentrate on the case where $I_{i_{1}, t}^{*}=I_{i_{2}, t}^{*}=1$. Recall the definition 
of $p_{j, t}\left(\boldsymbol{I}_{t}^{*}\right)$, we have

$$
\begin{aligned}
p_{j, t}\left(\boldsymbol{I}_{t}^{*}\right)= & \operatorname{Pr}\left(I_{j, t}^{*}=1, b_{j}+c_{j} \cdot\left[\tilde{D}-a_{j}\right]^{+} \leq \min _{\{0\} \cup\left\{i: I_{i, t}^{*}=1\right\}}\left\{b_{i}+c_{i} \cdot\left[\tilde{D}-a_{i}\right]^{+}\right\}\right) \\
\leq & \operatorname{Pr}\left(b_{j}+c_{j} \cdot\left[\tilde{D}-a_{j}\right]^{+} \leq \min _{\{0\} \cup\left\{i: I_{i, t}^{*}=1\right\}}\left\{b_{i}+c_{i} \cdot\left[\tilde{D}-a_{i}\right]^{+}\right\}\right) \\
\leq & \operatorname{Pr}\left(b_{j}+c_{j} \cdot\left[\tilde{D}-a_{j}\right]^{+} \leq b_{i_{1}}+c_{i_{1}} \cdot\left[\tilde{D}-a_{i_{1}}\right]^{+},\right. \\
& \left.\quad b_{j}+c_{j} \cdot\left[\tilde{D}-a_{j}\right]^{+} \leq b_{i_{2}}+c_{i_{2}} \cdot\left[\tilde{D}-a_{i_{2}}\right]^{+}\right) \\
= & \operatorname{Pr}\left(X_{i_{1}, j} \leq \tilde{D} \leq X_{j, i_{2}}\right) \\
= & F\left(X_{j, i_{2}}\right)-F\left(X_{i_{1}, j}\right) .
\end{aligned}
$$

So (27) holds. (28) discusses the case when plan $j$ is preferred over the reservation price (we define it as plan 0) and plan $i_{2}$, where $i_{2}>j$. (29) shows the case when plan $j$ is preferred to plan $i_{1}$, where $i_{1}<j$. (30) compares the value of plan $j$ with the reservation price and (31) discusses the situation when none of the plans is chosen by customers. We conclude that $p_{j, t}\left(\boldsymbol{I}_{t}^{*}\right)$ satisfies $(27)$ to $(31)$.

We construct $\boldsymbol{p} \boldsymbol{p}^{*}$ as $p p_{j, t}^{*}=\sum_{\boldsymbol{I}_{t} \in\{0,1\}^{J}} p_{j, t}\left(\boldsymbol{I}_{t}\right) \cdot K_{\boldsymbol{I}_{t}, t}^{*} \cdot \pi_{0, t}^{*}$. By definition of $\boldsymbol{I}_{t}^{*}$, $\boldsymbol{p} \boldsymbol{p}^{*}$ becomes $p p_{j, t}^{*}=p_{j, t}\left(\boldsymbol{I}_{t}^{*}\right) \cdot \pi_{0, t}^{*}$. Because $p_{j, t}\left(\boldsymbol{I}_{t}^{*}\right)$ satisfies $(27)$ to $(31)$, we know that $p p_{j, t}^{*}$ satisfies constraints $(\mathrm{PP})$ in $(\mathrm{C})$. Because $\beta_{j, t}^{*}=p_{j, t}\left(\boldsymbol{I}_{t}^{*}\right) \cdot \pi_{0, t}^{*}$, we have $\beta_{j, t}^{*}=p p_{j, t}^{*}$. When $I_{j, t}^{*}=1, \beta_{j, t}^{*}=p p_{j, t}^{*}$. When $I_{j, t}^{*}=0, \beta_{j, t}^{*}=0$. So $\beta_{j, t}^{*}$ satisfies

$$
p p_{j, t}^{*}-M \cdot\left(1-I_{j, t}^{*}\right) \leq \beta_{j, t} \leq p p_{j, t}^{*}+M \cdot\left(1-I_{j, t}^{*}\right)
$$

(24) and (32) suggest that $\boldsymbol{\beta}^{*}$ satisfies constraints (JP') in (C). We can conclude that $\left(\boldsymbol{\pi}^{*}, \boldsymbol{L}^{*}, \boldsymbol{\beta}^{*}, \boldsymbol{\gamma}^{*}, \boldsymbol{I}^{*}, \boldsymbol{p} \boldsymbol{p}^{*}\right)$ satisfies all the constraints in (C). So $\left(\boldsymbol{\pi}^{*}, \boldsymbol{L}^{*}, \boldsymbol{\beta}^{*}, \boldsymbol{\gamma}^{*}, \boldsymbol{I}^{*}, \boldsymbol{p} \boldsymbol{p}^{*}\right)$ is a feasible solution to $(\mathrm{C})$.

Step 2. $\hat{z_{p}}(\overline{\boldsymbol{\pi}}) \geq z_{p}(\overline{\boldsymbol{\pi}})$. 
Because $\left(\boldsymbol{\pi}^{*}, \boldsymbol{L}^{*}, \boldsymbol{\beta}^{*}, \boldsymbol{\gamma}^{*}, \boldsymbol{I}^{*}, \boldsymbol{p} \boldsymbol{p}^{*}\right)$ is a feasible solution to $(\mathrm{C})$,

$$
\begin{aligned}
\hat{z}_{p}(\overline{\boldsymbol{\pi}}) \geq & \sum_{t=1}^{T} \sum_{j=1}^{J} \pi_{j, t}^{*} \cdot \mathbb{E}\left\{b_{j}+c_{j} \cdot\left[\tilde{D}-a_{j}\right]^{+}\right\}+\sum_{t=1}^{T-1} \sum_{j=1}^{J} \beta_{j, t}^{*} \cdot \mathbb{E}\left\{b_{j}+c_{j} \cdot\left[\tilde{D}-a_{j}\right]^{+}\right\} \\
= & \sum_{t=1}^{T} \sum_{j=1}^{J} \pi_{j, t}^{*} \cdot \mathbb{E}\left\{b_{j}+c_{j} \cdot\left[\tilde{D}-a_{j}\right]^{+}\right\} \\
& \left.\quad+\sum_{t=1}^{T-1} \sum_{j=1}^{J} p_{j, t}\left(\boldsymbol{I}_{t}^{*}\right) \cdot \pi_{0, t}^{*} \cdot \mathbb{E}\left\{b_{j}+c_{j} \cdot\left[\tilde{D}-a_{j}\right]^{+}\right\} \quad \text { (by definition of } \beta_{j, t}^{*}\right) \\
= & \sum_{t=1}^{T} \sum_{j=1}^{J} \pi_{j, t}^{*} \cdot \mathbb{E}\left\{b_{j}+c_{j} \cdot\left[\tilde{D}-a_{j}\right]^{+}\right\} \\
& +\sum_{t=1}^{T-1} \pi_{0, t}^{*} \sum_{\boldsymbol{I}_{t} \in\{0,1\}^{j}} p_{j, t}\left(\boldsymbol{I}_{t}\right) \cdot K_{\boldsymbol{I}_{t}, t}^{*} \cdot \mathbb{E}\left\{b_{j}+c_{j} \cdot\left[\tilde{D}-a_{j}\right]^{+}\right\}
\end{aligned}
$$

(by definition of $\boldsymbol{I}_{t}^{*}$ )

$$
\begin{aligned}
&=\sum_{t=1}^{T} \sum_{j=1}^{J} \pi_{j, t}^{*} \cdot \mathbb{E}\left\{b_{j}+c_{j} \cdot\left[\tilde{D}-a_{j}\right]^{+}\right\} \\
&+\sum_{t=1}^{T-1} \sum_{\boldsymbol{I}_{t} \in\{0,1\}^{j}} p_{j, t}\left(\boldsymbol{I}_{t}\right) \cdot \Pi_{\boldsymbol{I}_{t}, t}^{*} \cdot \mathbb{E}\left\{b_{j}+c_{j} \cdot\left[\tilde{D}-a_{j}\right]^{+}\right\} \\
&\left(\Pi_{\boldsymbol{I}_{t}, t}^{*}=K_{\boldsymbol{I}_{t}, t}^{*} \cdot \pi_{0, t}^{*}, \forall \boldsymbol{I}_{t} \in\{0,1\}^{J}\right)
\end{aligned}
$$

$$
=z_{p}(\overline{\boldsymbol{\pi}}) . \quad\left(\left(\boldsymbol{\pi}^{*}, \boldsymbol{L}^{*}, \boldsymbol{K}_{\boldsymbol{I}}^{*}, \boldsymbol{\Pi}_{\boldsymbol{I}}^{*}\right) \text { is the optimal solution to }(\mathrm{B})\right)
$$

Step 3. Given that $(\hat{\boldsymbol{\pi}}, \hat{\boldsymbol{L}}, \hat{\boldsymbol{\beta}}, \hat{\boldsymbol{\gamma}}, \hat{\boldsymbol{I}}, \hat{\boldsymbol{p p}})$ is the optimal solution to (C), there exist $\hat{\boldsymbol{K}}_{\boldsymbol{I}}$, and $\hat{\boldsymbol{\Pi}}_{\boldsymbol{I}}$ such that $\left(\hat{\boldsymbol{\pi}}, \hat{\boldsymbol{L}}, \hat{\boldsymbol{K}}_{\boldsymbol{I}}, \hat{\boldsymbol{\Pi}}_{\boldsymbol{I}}\right)$ is a feasible solution to $(\mathrm{B})$.

We define $(\hat{\boldsymbol{\pi}}, \hat{\boldsymbol{L}}, \hat{\boldsymbol{\beta}}, \hat{\gamma}, \hat{\boldsymbol{I}}, \hat{\boldsymbol{p} p})$ as the unique optimal solution or one of the optimal solutions to (C). Apparently $(\hat{\boldsymbol{\pi}}, \hat{\boldsymbol{L}}, \hat{\boldsymbol{\beta}}, \hat{\boldsymbol{\gamma}}, \hat{\boldsymbol{I}}, \hat{\boldsymbol{p} p})$ is also a feasible solution to (C) and satisfies

$$
\begin{aligned}
& \hat{\pi}_{j, t} \cdot \bar{\theta}_{j}-M \cdot\left(1-\hat{L}_{t}\right) \leq \hat{\gamma}_{j, t} \leq \hat{\pi}_{j, t} \cdot \bar{\theta}_{j}+M \cdot\left(1-\hat{L}_{t}\right), \\
& -M \cdot \hat{L}_{t} \leq \hat{\gamma}_{j, t} \leq M \cdot \hat{L}_{t} \\
& -M \cdot\left(1-\hat{L}_{t}\right) \leq \sum_{j=1}^{J} \alpha_{j} \cdot \hat{\pi}_{j, t}-\Omega \leq M \cdot \hat{L}_{t}, \\
& \hat{L}_{t} \in\{0,1\} .
\end{aligned}
$$


Equations (33), (34), (35) and (36) suggest that if $\sum_{j=1}^{J} \alpha_{j} \cdot \hat{\pi}_{j, t} \geq \Omega$, then $\hat{L}_{t}=1$ and $\hat{\gamma}_{j, t}=\hat{\pi}_{j, t} \cdot \bar{\theta}_{j}$. Meanwhile, $\sum_{j=1}^{J} \alpha_{j} \cdot \hat{\pi}_{j, t}<\Omega$, then $\hat{L}_{t}=0$ and $\hat{\gamma}_{j, t}=0$. Because

$$
\theta_{j, t}\left(\hat{\boldsymbol{\pi}}_{t}\right)= \begin{cases}\bar{\theta}_{j}, & \text { if } \sum_{j=1}^{J} \alpha_{j} \cdot \hat{\pi}_{j, t} \geq \Omega, \\ 0, & \text { if } \sum_{j=1}^{J} \alpha_{j} \cdot \hat{\pi}_{j, t}<\Omega .\end{cases}
$$

we conclude that $\hat{\gamma}_{j, t}=\hat{\pi}_{j, t} \cdot \theta_{j, t}\left(\hat{\pi}_{t}\right)$.

Variables $\hat{\beta}_{j, t}$ and $\hat{p}_{j, t}$ satisfy the following constraints:

$$
\begin{aligned}
& -M \cdot \hat{I}_{j, t} \leq \beta_{j, t} \leq M \cdot \hat{I}_{j, t} \\
& p p_{j, t}-M \cdot\left(1-\hat{I}_{j, t}\right) \leq \beta_{j, t} \leq p p_{j, t}+M \cdot\left(1-\hat{I}_{j, t}\right) \\
& p p_{j, t} \leq M \cdot \hat{I}_{j, t} \\
& \sum_{j=0}^{J} p p_{j, t}=\hat{\pi}_{0, t} \\
& p p_{j, t} \leq \hat{\pi}_{0, t} \cdot\left[F\left(X_{j, i_{2}}\right)-F\left(X_{i_{1}, j}\right)\right]+M \cdot\left(2-\hat{I}_{i_{1}, t}-\hat{I}_{i_{2}, t}\right), \forall 1 \leq i_{1}<j<i_{2} \leq J \\
& p p_{j, t} \leq \hat{\pi}_{0, t} \cdot\left[F\left(X_{j, i_{2}}\right)-F\left(X_{0, j}\right)\right]+M \cdot\left(1-\hat{I}_{i_{2}, t}\right), \forall 1 \leq j<i_{2} \leq J \\
& p p_{j, t} \leq \hat{\pi}_{0, t} \cdot\left[1-F\left(X_{i_{1}, j}\right)\right]+M \cdot\left(1-\hat{I}_{i_{1}, t}\right), \forall 1 \leq i_{1}<j \leq J \\
& p p_{j, t} \leq \hat{\pi}_{0, t} \cdot\left[1-F\left(X_{0, j}\right)\right]+M \cdot\left(1-\hat{I}_{j, t}\right), \forall 1 \leq j \leq J \\
& p p_{0, t} \leq \hat{\pi}_{0, t} \cdot F\left(X_{0, j}\right)+M \cdot\left(1-\hat{I}_{j, t}\right), \forall 1 \leq j \leq J
\end{aligned}
$$

(39), (41)-(45) infer that

$$
\begin{aligned}
& \hat{p p} p_{j, t} \leq \hat{\pi}_{0, t} . \\
& \min _{\substack{i_{1}: \hat{I}_{1, t}=1 \\
i_{2}: i_{i_{2}, t}=1}}\left\{F\left(X_{j, i_{2}}\right)-F\left(X_{i_{1}, j}\right), F\left(X_{j, i_{2}}\right)-F\left(X_{0, j}\right), 1-F\left(X_{i_{1}, j}\right), 1-F\left(X_{0, j}\right)\right\}, \\
& \hat{p p}_{0, t} \leq \hat{\pi}_{0, t} \cdot \min _{\left\{j: \hat{I}_{j, t}=1\right\}}\left\{F\left(X_{0, j}\right)\right\} . \\
& \text { (if } \hat{I}_{j, t}=1 \text { ) }
\end{aligned}
$$

Moreover,

$$
\hat{p p}_{j, t}=0 \text {. }
$$$$
\text { (if } \hat{I}_{j, t}=0 \text { ) }
$$ 
For given $\hat{\boldsymbol{I}}_{t}$, by definition of $p_{j, t}\left(\boldsymbol{I}_{t}\right)$,

$$
\begin{aligned}
p_{j, t}\left(\hat{\boldsymbol{I}}_{t}\right) & =\operatorname{Pr}\left(\hat{I}_{j, t}=1, b_{j}+c_{j} \cdot\left[\tilde{D}-a_{j}\right]^{+} \leq \min _{\{0\} \cup\left\{i: I_{i}=1\right\}}\left\{b_{i}+c_{i} \cdot\left[\tilde{D}-a_{i}\right]^{+}\right\}\right) \\
& =\hat{I}_{j, t} \cdot \\
& \min _{\substack{i_{1}: \hat{I}_{1, t}=1 \\
i_{2}: i_{i_{2}, t}=1}}\left\{F\left(X_{j, i_{2}}\right)-F\left(X_{i_{1}, j}\right), F\left(X_{j, i_{2}}\right)-F\left(X_{0, j}\right), 1-F\left(X_{i_{1}, j}\right), 1-F\left(X_{0, j}\right)\right\} \\
p_{0, t}\left(\hat{\boldsymbol{I}}_{t}\right) & =\operatorname{Pr}\left(b_{0}+c_{0} \cdot\left[\tilde{D}-a_{0}\right]^{+} \leq \min _{\left\{i: I_{i}=1\right\}}\left\{b_{i}+c_{i} \cdot\left[\tilde{D}-a_{i}\right]^{+}\right\}\right) \\
& =\min _{\left\{i: I_{i}=1\right\}}\left\{F\left(X_{0, i}\right)\right\} .
\end{aligned}
$$

Because $\sum_{j=0}^{J} p_{j, t}\left(\hat{\boldsymbol{I}}_{t}\right)=1$, summing up all the equations in (46),

$$
\begin{aligned}
& \sum_{j=1}^{J} \hat{I}_{j, t} \min _{\substack{i_{1}: \hat{I}_{1}, t=1 \\
i_{2}: \hat{I}_{i_{2}, t}=1}}\left\{F\left(X_{j, i_{2}}\right)-F\left(X_{i_{1}, j}\right), F\left(X_{j, i_{2}}\right)-F\left(X_{0, j}\right), 1-F\left(X_{i_{1}, j}\right), 1-F\left(X_{0, j}\right)\right\} \\
& \quad+\min _{\left\{i: I_{i}=1\right\}}\left\{F\left(X_{0, i}\right)\right\} \\
& \quad=1 .
\end{aligned}
$$

To conclude, we have $\sum_{j=0}^{J} \hat{p} p_{j, t} \leq \hat{\pi}_{0, t}$. Combined with (40),

$$
\begin{aligned}
\hat{p p_{j, t}}= & \hat{\pi}_{0, t} \cdot \\
& \min _{\substack{i_{1}: \hat{I}_{1}, t=1 \\
i_{2}: \hat{I}_{i_{2}, t}=1}}\left\{F\left(X_{j, i_{2}}\right)-F\left(X_{i_{1}, j}\right), F\left(X_{j, i_{2}}\right)-F\left(X_{0, j}\right), 1-F\left(X_{i_{1}, j}\right), 1-F\left(X_{0, j}\right)\right\}, \\
\hat{p p_{0, t}}= & \hat{\pi}_{0, t} \cdot \min _{\left\{j: I_{j}=1\right\}}\left\{F\left(X_{0, j}\right)\right\} .
\end{aligned}
$$

By definition of $p_{j, t}\left(\hat{\boldsymbol{I}}_{t}\right)$, we have $\hat{p} p_{j, t}=\hat{\pi}_{0, t} \cdot p_{j, t}\left(\hat{\boldsymbol{I}}_{t}\right)$. Because of (37) and (38), we know that

$$
\hat{\beta}_{j, t}=\hat{\pi}_{0, t} \cdot p_{j, t}\left(\hat{\boldsymbol{I}}_{t}\right)
$$


If $\sum_{j=1}^{J} \alpha_{j} \cdot \hat{\pi}_{j, t} \geq \Omega$, we have

$$
\begin{aligned}
\hat{\pi}_{j, t+1} & =\hat{\pi}_{j, t}+\hat{\beta}_{j, t}-\hat{\gamma}_{j, t} \\
& =\hat{\pi}_{j, t}+\hat{\pi}_{0, t} \cdot p_{j, t}\left(\hat{\boldsymbol{I}}_{t}\right)-\hat{\pi}_{j, t} \cdot \bar{\theta}_{j} \\
& =\hat{\pi}_{0, t} \cdot p_{j, t}\left(\hat{\boldsymbol{I}}_{t}\right)+\left(1-\bar{\theta}_{j}\right) \cdot \hat{\pi}_{j, t} \\
& \left.=S\left(\hat{\boldsymbol{I}}_{t}, 1\right)_{j, 0} \cdot \hat{\pi}_{j, t}+S\left(\hat{\boldsymbol{I}}_{t}, 1\right)_{j, j} \cdot \hat{\pi}_{j, t}, \quad \text { (by definition of } S\left(\hat{\boldsymbol{I}}_{t}, 1\right)\right) \\
\hat{\pi}_{0, t+1} & =\hat{\pi}_{0, t}-\sum_{j=1}^{J} \hat{\beta}_{j, t}+\sum_{j=1}^{J} \hat{\gamma}_{j, t} \\
& =\hat{\pi}_{0, t}-\sum_{j=1}^{J} \hat{\pi}_{0, t} \cdot p_{j, t}\left(\hat{\boldsymbol{I}}_{t}\right)+\sum_{j=1}^{J} \hat{\pi}_{j, t} \cdot \bar{\theta}_{j} \\
& =\hat{\pi}_{0, t} \cdot\left(1-\sum_{j=1}^{J} p_{j, t}\left(\hat{\boldsymbol{I}}_{t}\right)\right)+\sum_{j=1}^{J} \hat{\pi}_{j, t} \cdot \bar{\theta}_{j} \\
& \left.=S\left(\hat{\boldsymbol{I}}_{t}, 1\right)_{0,0} \cdot \hat{\pi}_{0, t}+\sum_{j=1}^{J} S\left(\hat{\boldsymbol{I}}_{t}, 1\right)_{0, j} \cdot \hat{\pi}_{j, t} \text {. (by definition of } S\left(\boldsymbol{I}_{t}, 1\right)\right)
\end{aligned}
$$

Equation (48) suggests that $\hat{\boldsymbol{\pi}}_{t+1}=S\left(\boldsymbol{I}_{t}, 1\right) \cdot \hat{\boldsymbol{\pi}}_{t}$. If $\sum_{j=1}^{J} \alpha_{j} \cdot \hat{\pi}_{j, t}<\Omega$, similarly, there exists $\hat{\boldsymbol{\pi}}_{t+1}=S\left(\hat{\boldsymbol{I}}_{t}, 0\right) \cdot \hat{\boldsymbol{\pi}}_{t}$. We infer that $(\hat{\boldsymbol{\pi}}, \hat{\boldsymbol{I}}, \hat{\boldsymbol{L}})$ is a feasible solution to

$$
\begin{aligned}
& \boldsymbol{\pi}_{t+1} \geq S\left(\boldsymbol{I}_{t}, 1\right) \cdot \boldsymbol{\pi}_{t}-M \cdot\left(1-L_{t}\right), \\
& \boldsymbol{\pi}_{t+1} \geq S\left(\boldsymbol{I}_{t}, 0\right) \cdot \boldsymbol{\pi}_{t}-M \cdot L_{t} .
\end{aligned}
$$

We construct $\hat{K}_{\boldsymbol{I}_{t}, t}$ as follows. For all $\boldsymbol{I}_{t} \in\{0,1\}^{J}$, if $\boldsymbol{I}_{t}=\hat{\boldsymbol{I}}_{t}, \hat{K}_{\boldsymbol{I}_{t}, t}=1$. Otherwise, $\hat{K}_{\boldsymbol{I}_{t}, t}=0$. We also construct $\hat{\Pi}_{\boldsymbol{I}_{t}, t}$ as $\hat{\Pi}_{\boldsymbol{I}_{t}, t}=\hat{\pi}_{0, t} \cdot \hat{K}_{\boldsymbol{I}_{t}, t}, \forall \boldsymbol{I}_{t} \in\{0,1\}^{J}$. Further we get rid of $\hat{\boldsymbol{I}}_{t}$ in (49) and find that $\left(\hat{\boldsymbol{\pi}}, \hat{\boldsymbol{L}}, \hat{\boldsymbol{K}}_{\boldsymbol{I}}, \hat{\boldsymbol{\Pi}}_{\boldsymbol{I}}\right)$ is a feasible solution to

$$
\begin{aligned}
& \boldsymbol{\pi}_{t+1} \geq S\left(\boldsymbol{I}_{t}, 1\right) \cdot \boldsymbol{\pi}_{t}-M \cdot\left(1-L_{t}\right)-M \cdot\left(1-K_{\boldsymbol{I}_{t}, t}\right), \forall \boldsymbol{I}_{t} \in\{0,1\}^{J}, \\
& \boldsymbol{\pi}_{t+1} \geq S\left(\boldsymbol{I}_{t}, 0\right) \cdot \boldsymbol{\pi}_{t}-M \cdot L_{t}-M \cdot\left(1-K_{\boldsymbol{I}_{t}, t}\right), \forall \boldsymbol{I}_{t} \in\{0,1\}^{J}, \\
& \quad \sum_{\boldsymbol{I}_{t} \in\{0,1\}^{J}} K_{\boldsymbol{I}_{t}, t}=1, \quad K_{\boldsymbol{I}_{t}, t} \in\{0,1\}, \forall \boldsymbol{I}_{t} \in\{0,1\}^{J}, \\
& \prod_{\boldsymbol{I}_{t}, t} \leq \pi_{0, t}, \quad \Pi_{\boldsymbol{I}_{t}, t} \leq K_{\boldsymbol{I}_{t}, t}, \forall \boldsymbol{I}_{t} \in\{0,1\}^{J} .
\end{aligned}
$$

We conclude that $\left(\hat{\boldsymbol{\pi}}, \hat{\boldsymbol{L}}, \hat{\boldsymbol{K}}_{\boldsymbol{I}}, \hat{\boldsymbol{\Pi}}_{\boldsymbol{I}}\right)$ satisfies the constraints in $(\mathrm{B})$, so $\left(\hat{\boldsymbol{\pi}}, \hat{\boldsymbol{L}}, \hat{\boldsymbol{K}}_{\boldsymbol{I}}, \hat{\boldsymbol{\Pi}}_{\boldsymbol{I}}\right)$ is a feasible solution to $(\mathrm{B})$. 
Step 4. $z_{p}(\overline{\boldsymbol{\pi}}) \geq \hat{z}_{p}(\overline{\boldsymbol{\pi}})$.

Because $\left(\hat{\boldsymbol{\pi}}, \hat{\boldsymbol{L}}, \hat{\boldsymbol{K}}_{\boldsymbol{I}}, \hat{\boldsymbol{\Pi}}_{\boldsymbol{I}}\right)$ is a feasible solution to (B),

$$
\begin{aligned}
z_{p}(\overline{\boldsymbol{\pi}}) \geq & \sum_{t=1}^{T} \sum_{j=1}^{J} \hat{\pi}_{j, t} \cdot \mathbb{E}\left\{b_{j}+c_{j} \cdot\left[\tilde{D}-a_{j}\right]^{+}\right\} \\
& +\sum_{t=1}^{T-1} \sum_{j=1}^{J} \sum_{\boldsymbol{I}_{t} \in\{0,1\}^{J}} p_{j, t}\left(\boldsymbol{I}_{t}\right) \cdot \hat{\Pi}_{\boldsymbol{I}_{t}, t} \cdot \mathbb{E}\left\{b_{j}+c_{j} \cdot\left[\tilde{D}-a_{j}\right]^{+}\right\} \\
= & \sum_{t=1}^{T} \sum_{j=1}^{J} \hat{\pi}_{j, t} \cdot \mathbb{E}\left\{b_{j}+c_{j} \cdot\left[\tilde{D}-a_{j}\right]^{+}\right\} \\
& +\sum_{t=1}^{T-1} \sum_{j=1}^{J} p_{j, t}\left(\hat{\boldsymbol{I}}_{t}\right) \cdot \hat{\pi}_{0, t} \cdot \mathbb{E}\left\{b_{j}+c_{j} \cdot\left[\tilde{D}-a_{j}\right]^{+}\right\}
\end{aligned}
$$

(by definition of $\hat{\Pi}_{\boldsymbol{I}_{t}, t}, \hat{K}_{\boldsymbol{I}_{t}, t}$ )

$$
=\sum_{t=1}^{T} \sum_{j=1}^{J} \hat{\pi}_{j, t} \cdot \mathbb{E}\left\{b_{j}+c_{j} \cdot\left[\tilde{D}-a_{j}\right]^{+}\right\}+\sum_{t=1}^{T-1} \sum_{j=1}^{J} \hat{\beta}_{j, t} \cdot \mathbb{E}\left\{b_{j}+c_{j} \cdot\left[\tilde{D}-a_{j}\right]^{+}\right\}
$$

(by equation (47))

$$
=\hat{z}_{p}(\overline{\boldsymbol{\pi}}) . \quad((\hat{\boldsymbol{\pi}}, \hat{\boldsymbol{L}}, \hat{\boldsymbol{\beta}}, \hat{\boldsymbol{\gamma}}, \hat{\boldsymbol{I}}, \hat{\boldsymbol{p} p}) \text { is the optimal solution to }(\mathrm{C}))
$$

In conclusion, we have shown $z_{p}(\overline{\boldsymbol{\pi}})=\hat{z}_{p}(\overline{\boldsymbol{\pi}})$. Because Proposition 2 suggests $z_{p}(\overline{\boldsymbol{\pi}})=\bar{V}_{1}(\overline{\boldsymbol{\pi}}), \hat{z}_{p}(\overline{\boldsymbol{\pi}})=z_{p}(\overline{\boldsymbol{\pi}})=\bar{V}_{1}(\overline{\boldsymbol{\pi}})$. So Proposition 3 holds. 
Table 8: Major notation and symbols (exogenous parameters)

\section{Exogenous parameters}

$C=$ The capacity of network that provided by the SP

$N=$ Total number of customers in the market

$J=$ Total number of cell phone plans

$\tilde{D}=$ The data usage of a customer

$a_{j}=$ Plan $j$ 's amount of allotted data

$b_{j}=$ Plan j's monthly charge

$c_{j}=$ Plan $j$ 's overage per unit charge for data usage in excess of the allowance

$\boldsymbol{X}_{t}=\left(X_{1, t}, X_{2, t}, \ldots, X_{j, t}, \ldots, X_{J, t}\right)$, where $X_{j, t}$ denotes plan $j$ 's number of subscribed customers at the beginning of period $t$.

$\tilde{\boldsymbol{Y}}_{t}=\left(\tilde{Y}_{1, t}, \tilde{Y}_{2, t}, \ldots, \tilde{Y}_{j, t}, \ldots, \tilde{Y}_{J, t}\right)$, where $\tilde{Y}_{j, t}$ denotes the number of new customers subscribing plan $j$ in period $t$.

$\tilde{\boldsymbol{L}}_{t}=\left(\tilde{l}_{1, t}, \tilde{l}_{2, t}, \ldots, \tilde{l}_{j, t}, \ldots, \tilde{l}_{J, t}\right)$, where $\tilde{l}_{j, t}$ denotes the number of customers leaving plan $j$ at the end of period $t$.

$\boldsymbol{\pi}_{t}=\left(\pi_{0, t}, \pi_{1, t}, \ldots, \pi_{j, t}, \ldots, \pi_{J, t}\right)$, where $\pi_{0, t}$ denotes the pecentage of customers in potential pool and $\pi_{j, t}(1 \leq j \leq J)$ denotes the percentage of customers subscribed plan $j$ at the beginning of period $t$

$\overline{\boldsymbol{\pi}}=\left(\bar{\pi}_{0}, \bar{\pi}_{1}, \bar{\pi}_{2}, \ldots, \bar{\pi}_{j}, \ldots, \bar{\pi}_{J}\right)$, where $\bar{\pi}_{0}$ denotes the initial percentage of customers in potential pool and $\bar{\pi}_{j}(1 \leq j \leq J)$ denotes the initial percentage of customers subscribed plan $j$ 
Table 9: Major notation and symbols (decision variables and computed quantities)

\section{Decision variables}

$\boldsymbol{I}_{t}=\left(I_{1, t}, I_{2, t}, \ldots, I_{j, t}, \ldots, I_{J t},\right)$, where $I_{j, t}=1$ if plan $j$ is open in period $t ; I_{j, t}=0$ otherwise.

$V_{t}=$ Period $t$ 's value function (a.k.a. revenue-to-go function)

\section{Computed quantities}

$p_{j, t}=$ The probability that an unsubscribed customer subscribes plan $j$ in period $t$

$\theta_{j, t}=$ The probability that a plan $j$ 's subscribed customer quits the network in period $t$

$\alpha_{j}=$ Plan $j$ 's percentage of contribution to cause congestion

$\Omega=$ The threshold of network usage above which the network is congested

$\overline{V_{t}}=$ The average revenue per person when the customer population approaches positive infinity in period $t$

$L_{t}=$ Whether the system's total usage overrides the capacity in period $t . L_{t}=1$ denotes the total usage is larger than system capacity. $L_{t}=0$ describes the opposite situation

$K_{\boldsymbol{I}_{t}, t}=$ Whether control $\boldsymbol{I}_{t}$ is used in period $t . \quad K_{\boldsymbol{I}_{t}, t}=1$ denotes control $\boldsymbol{I}_{t}$ is used. $K_{\boldsymbol{I}_{t}, t}=0$ describes the opposite situation

$z_{p}(\overline{\boldsymbol{\pi}})=$ The optimal value of the SP's optimization problem when the initial state is $\overline{\boldsymbol{\pi}}$

$M=$ A very large number 


\section{References}

Ascarza, E., Lambrecht, A., Vilcassim, N., 2009. When talk is free: An analysis of subscriber behavior under two-and three-part tariffs. Tech. rep., Technical report, Working Paper, London Business School.

Bar-Gill, O., Stone, R., 2012. Pricing misperceptions: Explaining pricing structure in the cell phone service market. Journal of Empirical Legal Studies 9 (3), 430-456.

Basar, T., Srikant, R., 2002. Revenue-maximizing pricing and capacity expansion in a many-users regime. In: INFOCOM 2002. Twenty-First Annual Joint Conference of the IEEE Computer and Communications Societies. Proceedings. IEEE. Vol. 1. IEEE, pp. 294-301.

Bouslah, B., Gharbi, A., Pellerin, R., 2015. Integrated production, sampling quality control and maintenance of deteriorating production systems with aoql constraint. Omega.

Burnham, T. A., Frels, J. K., Mahajan, V., 2003. Consumer switching costs: a typology, antecedents, and consequences. Journal of the Academy of Marketing Science 31 (2), 109-126.

Danaher, P. J., 2002. Optimal pricing of new subscription services: Analysis of a market experiment. Marketing Science 21 (2), 119-138.

Defraeye, M., Van Nieuwenhuyse, I., 2016. Staffing and scheduling under nonstationary demand for service: A literature review. Omega 58, 4-25.

Fang, Y., Zhang, Y., 2002. Call admission control schemes and performance analysis in wireless mobile networks. Vehicular Technology, IEEE Transactions on 51 (2), $371-382$.

Garg, V., 2010. Wireless Communications \& Networking. Morgan Kaufmann. 
Gopalakrishnan, A., Iyengar, R., Meyer, R., 2012. Consumer dynamic usage allocation and learning under multi-part tariffs: Theory and empirical evidence. Available at SSRN 2152157.

Henderson, T., Crowcroft, J., Bhatti, S., 2001. Congestion pricing. paying your way in communication networks. Internet Computing, IEEE 5 (5), 85-89.

Ho, T. H., Zheng, Y.-S., 2004. Setting customer expectation in service delivery: An integrated marketing-operations perspective. Management Science 50 (4), 479488.

Iyengar, R., Ansari, A., Gupta, S., 2007. A model of consumer learning for service quality and usage. Journal of Marketing Research 44 (4), 529-544.

Kim, H.-S., Yoon, C.-H., 2004. Determinants of subscriber churn and customer loyalty in the korean mobile telephony market. Telecommunications Policy 28 (9), $751-765$.

Kim, M.-K., Park, M.-C., Jeong, D.-H., 2004. The effects of customer satisfaction and switching barrier on customer loyalty in korean mobile telecommunication services. Telecommunications policy 28 (2), 145-159.

Lambrecht, A., Seim, K., Skiera, B., 2007. Does uncertainty matter? consumer behavior under three-part tariffs. Marketing Science 26 (5), 698-710.

Lee, J., Lee, J., Feick, L., 2001. The impact of switching costs on the customer satisfaction-loyalty link: mobile phone service in france. Journal of services marketing 15 (1), 35-48.

Maglaras, C., Zeevi, A., 2003. Pricing and capacity sizing for systems with shared resources: Approximate solutions and scaling relations. Management Science 49 (8), $1018-1038$. 
Miravete, E. J., 2002. Estimating demand for local telephone service with asymmetric information and optional calling plans. The Review of Economic Studies 69 (4), 943-971.

Pae, J. H., Hyun, J. S., 2006. Technology advancement strategy on patronage decisions: the role of switching costs in high-technology markets. Omega 34 (1), $19-27$.

Rasti, M., Sharafat, A. R., Seyfe, B., 2009. Pareto-efficient and goal-driven power control in wireless networks: a game-theoretic approach with a novel pricing scheme. IEEE/ACM Transactions on Networking (TON) 17 (2), 556-569.

Saraydar, C. U., Mandayam, N. B., Goodman, D., 2002. Efficient power control via pricing in wireless data networks. Communications, IEEE Transactions on 50 (2), 291-303.

Teo, T. S., Liu, J., 2007. Consumer trust in e-commerce in the united states, singapore and china. Omega 35 (1), 22-38.

Tsai, W.-H., Hung, S.-J., 2009. Dynamic pricing and revenue management process in internet retailing under uncertainty: an integrated real options approach. Omega $37(2), 471-481$.

Van Ackere, A., Haxholdt, C., Larsen, E. R., 2013. Dynamic capacity adjustments with reactive customers. Omega 41 (4), 689-705. 